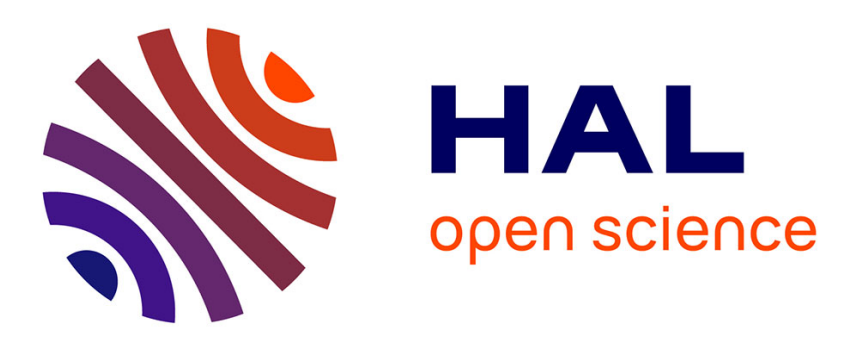

\title{
A micro-macro method for a kinetic graphene model in one-space dimension
}

Nicolas Crouseilles, Shi Jin, Mohammed Lemou, Florian Méhats

\section{To cite this version:}

Nicolas Crouseilles, Shi Jin, Mohammed Lemou, Florian Méhats. A micro-macro method for a kinetic graphene model in one-space dimension. Multiscale Modeling and Simulation: A SIAM Interdisciplinary Journal, 2020, 18 (1), pp.444-474. 10.1137/18M1173770 . hal-01883237

\section{HAL Id: hal-01883237 \\ https://hal.inria.fr/hal-01883237}

Submitted on 27 Sep 2018

HAL is a multi-disciplinary open access archive for the deposit and dissemination of scientific research documents, whether they are published or not. The documents may come from teaching and research institutions in France or abroad, or from public or private research centers.
L'archive ouverte pluridisciplinaire HAL, est destinée au dépôt et à la diffusion de documents scientifiques de niveau recherche, publiés ou non, émanant des établissements d'enseignement et de recherche français ou étrangers, des laboratoires publics ou privés. 


\title{
A MICRO-MACRO METHOD FOR A KINETIC GRAPHENE MODEL IN ONE-SPACE DIMENSION
}

\author{
NICOLAS CROUSEILLES, SHI JIN, MOHAMMED LEMOU, AND FLORIAN MÉHATS
}

\begin{abstract}
In this paper, for the one space dimensional semiclassical kinetic graphene model introduced in [20, we propose a micro-macro decomposition based numerical approach, which reduces the computational dimension of the nonlinear geometric optics method based numerical method for highly oscillatory transport equation developed in [6]. The method solves the highly oscillatory model in the original coordinate, yet can capture numerically the oscillatory space-time quantum solution pointwisely even without numerically resolving the frequency. We prove that the underlying micro-macro equations have smooth (up to certain order of derivatives) solutions with respect to the frequency, and then prove the uniform accuracy of the numerical discretization for a scalar model equation exhibiting the same oscillatory behavior. Numerical experiments verify the theory.
\end{abstract}

\section{Introduction}

Graphene is a two-dimensional flat monolayer of carbon atoms that displays unusual and intriguingelectronic properties arising from the bi-conically shaped Fermi surfaces near the Brillouin zone corners called Dirac points. In this material, the electrons propagate as massless Dirac Fermions travellig with the Fermi velocity $v_{F}$, which is 300 times smaller than the speed of light $v_{F} \approx \frac{c}{300} \approx 10^{6} \mathrm{~m} \cdot \mathrm{s}^{-1}$, and their behavior reproduces the physics of quantum electrodynamics but at much smaller energy scale. After the work of [22] where the graphene was produced for the first time, one has seen vast amount of works on this material, including applications in carbon-based electronic devices [18, mathematical analysis [11, 12], and numerical simulations, see e.g. [13] and references therein.

We are interested in the description of the transport of electrons in a single graphene layer, which is described by a two-dimensional Dirac equation ([1, 3, 21]) of a graphene sheet in the presence of an external potential. This model consists of a small parameter $\hbar$ directly related to the Planck constant, thus its solution is highly oscillatory and computationally too expensive. Semiclassical methods, based on asymptotic models valid for small $\hbar$, are often more afforable for computation [15]. In this paper, we study such a model, developed in [20]. Unlike a typical geometric optics type approach, this model, based on approximation using the Wigner transform and Weyl quantization, evolves the entire Wigner matrix thus retains the off-diagonal entries of the Wigner matrix responsible for interband quantum transition information such as Berry connection and Berry phase, in addition to the Landau-Zener transition probability. In particular, we are interested in efficient numerical approximation method for this asymptotic model, aimed at developing a numerical method uniformly accurate in all frequencies-it can obtain 
accurate pointwise numerical solution- even without resolving numerically the high frequency.

Our approach begins with the nonlinear geometric optics (NGO) based method introduced in our previous work [6] which achieves a uniform numerical accuracy by combining a nonlinear geometric optics ansatz, which introduces an extra independent variable representing the oscillatory phase, and a Chapman-Enskog expansion induced initial data such as the resulting NGO equations give non-oscillatory solutions (to certain order of derivatives) in space and time. While this method can be directly applied to the kinetic model under study in this article, and even with random band gap [7, the aim of this paper is to further reduce the computational cost of this NGO method via the micro-macro decomposition, which has found success in multiscale kinetic simulations [2, 17, 19]. Note that this approach has been recently introduced in [5] for highly oscillatory (in time) systems. This allows us to design a numerical scheme on the original kinetic coordinate-without the extra dimension for the phase- which can still capture the space-time oscillatory solution pointwise even without numerical resolving the high frequency. Comparing with previous surface hopping based numerical method [10] (see a related model and mathematical study in [16]), which does not need to resolve the oscillations numerically and can capture the Landau-Zener transition probability, our method, based on the semiclassical model of [20, gives more accurate quantum phase information.

The article is organized as follows. In section 2 we introduce the semiclassical kinetic model for graphene, introduced in [20]. The micro-macro decomposition based numerical method is introduced for the kinetic model in Section 3. We prove the boundedness of the solution (up to certain derivatives) in terms of the frequency in section 4. Numerical approximations, as well as the proof of the uniform numerical convergence in the frequency for the time discretization, are given in 5 . In 6 we conduct numerical experiments to verify the theoretical results and numerical accuracy. The paper is concluded in section 7.

\section{A semi-classical model for graphene}

Consider the following kinetic model in two space dimension describing the transport of electrons in a graphene layer:

$$
\begin{aligned}
& \partial_{t} f_{ \pm} \pm \frac{p}{|p|} \cdot \nabla_{x} f_{ \pm}-\nabla_{x} V \cdot \nabla_{p} f_{ \pm}=\mp \frac{p^{\perp} \cdot \nabla_{x} V}{|p|^{3}} \Im\left(\left(p_{1}+i p_{2}\right) f_{i}\right) \\
& \partial_{t} f_{i}-\nabla_{x} V \cdot \nabla_{p} f_{i}=-\frac{2 i|p|}{\varepsilon} f_{i}+i \frac{p^{\perp} \cdot \nabla_{x} V}{|p|^{2}} f_{i}+\frac{i}{2} \frac{p^{\perp} \cdot \nabla_{x} V}{|p|^{3}}\left(p_{1}-i p_{2}\right)\left(f_{+}-f_{-}\right),
\end{aligned}
$$

where $x=\left(x_{1}, x_{2}\right), p=\left(p_{1}, p_{2}\right), p^{\perp}=\left(-p_{2}, p_{1}\right)$ and the unknowns are $f_{+}(t, x, p) \in$ $\mathbb{R}, f_{-}(t, x, p) \in \mathbb{R}$, the electron and hole Wigner functions, and $f_{i}(t, x, p) \in \mathbb{C}$ the off-diagonal part of the Wigner matrix. Here $V(t, x)$ is an external potential that modifies the crystal periodic potential. $\Im$ is the imaginary part.

The model (2.1)-(2.2) was derived in [20]. Let us recall the main lines of its derivation. The original quantum model, written in physical variables, is the following von Neumann equation

$$
i \hbar \partial_{t} \varrho=\left[v_{F} \mathcal{H}_{0}+e V \sigma_{0}, \varrho\right]
$$


where $\varrho$ is the density operator of the particles, $\sigma_{0}$ is the $2 \times 2$ identity matrix and the free Hamiltonian is $\mathcal{H}_{0}=A(\hbar D), D=-i \nabla_{x}, A$ being the matrix

$$
A(p)=\sigma \cdot p=\left(\begin{array}{cc}
0 & p_{1}-i p_{2} \\
p_{1}+i p_{2} & 0
\end{array}\right) .
$$

Let us write this system in dimensionless form. Introduce a characteristic length $L$ of the device, a characteristic energy $E$, a characteristic density $n$, choose the characteristic time $T=\frac{L}{v_{F}}$ and set

$$
x^{\prime}=\frac{x}{L}, \quad V^{\prime}=\frac{e V}{E}, \quad t^{\prime}=\frac{t}{T}, \quad \varrho^{\prime}=\frac{\varrho}{n L^{2}} .
$$

The von Neumann equation (2.3) reads as follows (dropping the' in the functions and the variables)

$$
i \varepsilon \partial_{t} \varrho=\left[A(\varepsilon D)+V \sigma_{0}, \varrho\right],
$$

where the dimensionless parameter $\varepsilon$ is given by

$$
\varepsilon=\frac{\hbar v_{F}}{E L} \text {. }
$$

Then we introduce the following orthogonal and Hermitian matrix

$$
\Theta(p)=\frac{1}{\sqrt{2}}\left(\begin{array}{cc}
1 & \frac{p_{1}-i p_{2}}{|p|} \\
\frac{p_{1}+i p_{2}}{|p|} & -1
\end{array}\right)
$$

which diagonalizes $A(p)$ :

$$
\Theta(p) A(p) \Theta(p)=\Lambda(p)=\left(\begin{array}{cc}
|p| & 0 \\
0 & -|p|
\end{array}\right)
$$

and consider the associated density operator

$$
\widetilde{\varrho}=\Theta(\varepsilon D) \varrho \Theta(\varepsilon D) \text {. }
$$

The Wigner transform of a density operator $\varrho$ is defined by

$$
W(\varrho)=\int_{\mathbb{R}^{2}} \rho\left(x-\frac{\eta}{2}, x+\frac{\eta}{2}\right) e^{-i p \cdot \eta / \varepsilon} d \eta,
$$

where $\rho$ denotes the integral kernel of $\varrho$. The inverse Wigner transform of a symbol $w(t, x, p)$, referred to as the Weyl quantization, is the operator acting on $\phi(x)$ as

$$
W^{-1}(w) \psi(x)=\frac{1}{(2 \pi \varepsilon)^{2}} \int_{\mathbb{R}^{4}} w\left(\frac{x+y}{2}, p\right) \psi(y) e^{i p \cdot(x-y) / \varepsilon} d p d y .
$$

Introducing the Wigner function

$$
f=\left(\begin{array}{cc}
f_{+} & f_{i} \\
\hline f_{i} & f_{-}
\end{array}\right)=\frac{1}{(2 \pi \varepsilon)^{2}} W(\widetilde{\varrho}),
$$

the matrix $f(t, x, p)$ satisfies the following equation

$$
\partial_{t} f=\frac{1}{i \varepsilon}\left[\mathcal{U}^{\prime}+\Lambda(p), f\right]_{\star}
$$

where $[a, b]_{\star}=a \star b-b \star a$,

$$
\mathcal{U}^{\prime}(x, p)=\Theta(p) \star V(x) \star \Theta(p),
$$

and where $\star$ denotes the Moyal product

$$
a \star b=W\left(W^{-1}(a) W^{-1}(b)\right) .
$$


The Moyal product can be expanded formally as a series in $\varepsilon$

$$
a \star b(x, p)=\sum_{n=0}^{+\infty} \sum_{\alpha, \beta,|\alpha|+|\beta|=n}\left(\frac{i \varepsilon}{2}\right)^{n} \frac{(-1)^{|\beta|}}{\alpha ! \beta !} \partial_{x}^{\alpha} \partial_{p}^{\beta} a(x, p) \partial_{x}^{\beta} \partial_{p}^{\alpha} b(x, p),
$$

where $\alpha=\left(\alpha_{1}, \alpha_{2}\right) \in \mathbb{N}^{2}$ is a multi-index, $|\alpha|=\alpha_{1}+\alpha_{2}, \alpha !=\alpha_{1} ! \alpha_{2} !, \partial_{x}^{\alpha}=\partial_{x_{1}}^{\alpha_{1}} \partial_{x_{2}}^{\alpha_{2}}$ and similarly for $\beta$. Using the first terms of this serie, one obtains formally

$$
[\Lambda, f]_{\star}=[\Lambda, f]-\frac{i \varepsilon}{2}\left\{\nabla_{p} \Lambda, \nabla_{x} f\right\}+\mathcal{O}\left(\varepsilon^{2}\right),
$$

where $\{A, B\}=A B+B A$, and

$$
\left[\mathcal{U}^{\prime}, f\right]_{\star}=i \varepsilon \nabla_{x} V \cdot \nabla_{p} f+\frac{i \varepsilon}{2}\left[\left[\Theta, \nabla_{p} \Theta \cdot \nabla_{x} V\right], f\right]+\mathcal{O}\left(\varepsilon^{2}\right) .
$$

Inserting these expansions in (2.5) and dropping the $\mathcal{O}(\varepsilon)$ terms yields (2.1), 2.2).

\section{The micro-macro formulation of the semi-classical model in one space dimension}

In one space dimension (i.e. where all the quantities depend only on $x \in \mathbb{R}, t \geq 0$ and two-dimensional momentum space $\left.p=\left(p_{x}, p_{y}\right) \in \mathbb{R}^{2}\right)$, the model 2.1)-2.2 for graphene reads [20, 10]:

$$
\begin{aligned}
& \partial_{t} f^{+}+\frac{p_{x}}{|p|} \partial_{x} f^{+}-E \partial_{p_{x}} f^{+}=\frac{p_{y}}{|p|^{3}} E \Im\left(p f_{i}\right), \\
& \partial_{t} f^{-}-\frac{p_{x}}{|p|} \partial_{x} f^{-}-E \partial_{p_{x}} f^{-}=-\frac{p_{y}}{|p|^{3}} E \Im\left(p f_{i}\right), \\
& \partial_{t} f_{i}-E \partial_{p_{x}} f_{i}=-i \frac{2|p|}{\varepsilon} f_{i}-i \frac{p_{y}}{|p|^{2}} E f_{i}-\frac{i}{2} \frac{p_{y}}{|p|^{3}} E \bar{p}\left(f^{+}-f^{-}\right),
\end{aligned}
$$

where $E=\partial_{x} V \in \mathbb{R}$ given. With a slight abuse of notation, we shall identify the complex $p=p_{x}+i p_{y}$ to the element $p=\left(p_{x}, p_{y}\right) \in \mathbb{R}^{2}$. System (3.1) is supplemented with the following initial conditions

$$
\left(f^{+}(0, x, p), f^{-}(0, x, p), f_{i}(0, x, p)\right)=\left(f_{\text {in }}^{+}(x, p), f_{\text {in }}^{-}(x, p), f_{\text {in }}(x, p)\right) .
$$

Let us introduce a phase function $S(t, x, p)$ designed to follow the main oscillation of this model. We consider the phase $S(t, x, p)$ solution to

$$
\partial_{t} S-E \partial_{p_{x}} S=2|p|, \quad S(0, x, p)=0,
$$

and rewrite system 3.1 in terms of $f^{ \pm}$and $g(t, x, p)=\exp \left(i \frac{S(t, x, p)}{\varepsilon}\right) f_{i}(t, x, p)$ :

$$
\begin{aligned}
& \partial_{t} f^{+}+\frac{p_{x}}{|p|} \partial_{x} f^{+}-E \partial_{p_{x}} f^{+}=\frac{p_{y}}{|p|^{3}} E \Im\left(p e^{-i S / \varepsilon} g\right), \\
& \partial_{t} f^{-}-\frac{p_{x}}{|p|} \partial_{x} f^{-}-E \partial_{p_{x}} f^{-}=-\frac{p_{y}}{|p|^{3}} E \Im\left(p e^{-i S / \varepsilon} g\right), \\
& \partial_{t} g-E \partial_{p_{x}} g=-i \frac{p_{y}}{|p|^{2}} E g-\frac{i}{2} \frac{p_{y}}{|p|^{3}} E \bar{p}\left(f^{+}-f^{-}\right) e^{i S / \varepsilon} .
\end{aligned}
$$

In the following Proposition, we reformulate (3.4) into a micro-macro model whose solution is smooth (in a sense we will precise in the next section) with respect to $\varepsilon$. 
This micro-macro model will be discretized by a numerical scheme which enjoys the uniform accuracy with respect to $\varepsilon$. The Proposition is stated in the homogeneous case ( $E$ is contant) while the non homogeneous case $E(x)$ will be considered in Appendix A.

Proposition 3.1. [ Formal reformulation of (3.1)-(3.2) ]

System (3.1) with initial conditions (3.2) satisfied by $\left(f^{ \pm}, f_{i}\right)$ is equivalent to the following micro-macro system satisfied by $\left(F_{0}^{ \pm}, G_{0}, h^{ \pm}, h, S\right)$ :

the macro model for $\left(F_{0}^{ \pm}, G_{0}\right)$ reads

$$
\begin{aligned}
\partial_{t} F_{0}^{+}+\mathcal{T}^{+} F_{0}^{+} & =0 \\
\partial_{t} F_{0}^{-}+\mathcal{T}^{-} F_{0}^{-} & =0 \\
\partial_{t} G_{0}-E \partial_{p_{x}} G_{0} & =-i \frac{p_{y}}{|p|^{2}} E G_{0}-i \varepsilon \frac{p_{y}^{2} E^{2}}{4|p|^{5}} G_{0}
\end{aligned}
$$

with $\mathcal{T}^{ \pm}= \pm p_{x} /|p| \partial_{x}-E \partial_{p_{x}} ;$

the micro model for $\left(h^{ \pm}, h\right)$ reads

$$
\begin{aligned}
\left(\partial_{t}+\mathcal{T}^{+}\right) h^{+}= & R[h]-\frac{2 \varepsilon p_{x} E}{|p|^{3}} R\left[i G_{0}\right]+\frac{\varepsilon E}{2|p|} R\left[i G_{0}\right] \\
& -\frac{\varepsilon p_{y} E}{2|p|^{3}} R\left[G_{0}\right]\left(1+\frac{\varepsilon p_{y} E}{4|p|^{3}}\right)-\frac{\varepsilon p_{x}}{2|p|^{2}} R\left[i \partial_{x} G_{0}\right] \\
\left(\partial_{t}+\mathcal{T}^{-}\right) h^{-}= & -R[h]+\frac{2 \varepsilon p_{x} E}{|p|^{3}} R\left[i G_{0}\right]-\frac{\varepsilon E}{2|p|} R\left[i G_{0}\right] \\
& +\frac{\varepsilon p_{y} E}{2|p|^{3}} R\left[G_{0}\right]\left(1+\frac{\varepsilon p_{y} E}{4|p|^{3}}\right)-\frac{\varepsilon p_{x}}{2|p|^{2}} R\left[i \partial_{x} G_{0}\right] \\
\left(\partial_{t}-E \partial_{p_{x}}\right) h= & \frac{3 \varepsilon p_{x} p_{y} \bar{p} E^{2}}{4|p|^{6}} e^{i S / \varepsilon}\left(F_{0}^{+}-F_{0}^{-}\right)-\frac{i p_{y} E}{|p|^{2}} h-\frac{i p_{y} \bar{p} E}{2|p|^{3}} e^{i S / \varepsilon}\left(h^{+}-h^{-}\right) \\
& -\varepsilon \frac{i p_{y}^{2} \bar{p}^{2} E^{2}}{4|p|^{7}} e^{2 i S / \varepsilon} \bar{G}_{0}-\frac{\varepsilon p_{y} p_{x} \bar{p} E}{4|p|^{5}} \partial_{x}\left(F_{0}^{+}+F_{0}^{-}\right) e^{i S / \varepsilon}
\end{aligned}
$$

with $R[\cdot]=\frac{p_{y}}{|p|^{3}} E \Im\left(p e^{-i S / \varepsilon} \cdot\right)$;

the equation for the phase $S$ reads

$$
\partial_{t} S-E \partial_{p_{x}} S=2|p|
$$

the initial conditions for the micro-macro model (3.5)-(3.7)-(3.8) are

$$
\begin{aligned}
F^{ \pm}(0, x, p) & =f_{i n}^{ \pm}(x, p) \mp \frac{\varepsilon p_{y} E}{2|p|^{4}} \Im\left(i p f_{i n}(x, p)\right), \\
G_{0}(0, x, p) & =f_{i n}+\frac{\varepsilon p_{y} \bar{p} E}{4|p|^{4}}\left(f_{i n}^{+}(x, p)-f_{i n}^{-}(x, p)\right), \\
h^{ \pm}(0, x, p) & =\mp \varepsilon^{2} \frac{p_{y}^{2} E^{2}}{8|p|^{6}}\left(f_{i n}^{+}(x, p)-f_{i n}^{-}(x, p)\right), \\
h(0, x, p) & =-\varepsilon^{2} \frac{p_{y}^{2} \bar{p} E^{2}}{4|p|^{8}} \Im\left(i p f_{i n}(x, p)\right), \\
S(0, x, p) & =0 .
\end{aligned}
$$


Indeed, from the solution $\left(F_{0}^{ \pm}, G_{0}, h^{ \pm}, h, S\right)$ of the micro-macro model, we can reconstruct the solution $\left(f^{ \pm}, f_{i}\right)$ of the original model (3.1) as

$$
\begin{aligned}
f^{ \pm}(t, x, p) & =F_{0}^{ \pm}(t, x, p) \pm \frac{\varepsilon p_{y} E}{2|p|^{4}} \Im\left(i p e^{-i S / \varepsilon} G_{0}(t, x, p)\right)+h^{ \pm}(t, x, p), \\
f_{i}(t, x, p) & =e^{-i S / \varepsilon} G_{0}(t, x, p)-\frac{\varepsilon p_{y} \bar{p} E}{4|p|^{4}}\left(F_{0}^{+}(t, x, p)-F_{0}^{-}(t, x, p)\right)+e^{-i S / \varepsilon} h(t, x, p) .
\end{aligned}
$$

Proof. First, as in [6], we introduce the augmented unknowns $\left(F^{ \pm}, G\right)(t, x, p, \tau)$ satisfying

$$
f^{ \pm}(t, x, p)=F^{ \pm}\left(t, x, p, \frac{S(t, x, p)}{\varepsilon}\right), \quad g(t, x, p)=G\left(t, x, p, \frac{S(t, x, p)}{\varepsilon}\right),
$$

with $f^{ \pm}, g$ solutions of (3.4). One then has:

$$
\begin{aligned}
& \partial_{t} F^{+}+\frac{p_{x}}{|p|} \partial_{x} F^{+}-E \partial_{p_{x}} F^{+}=-\frac{2|p|}{\varepsilon} \partial_{\tau} F^{+}+\frac{p_{y}}{|p|^{3}} E \Im\left(p e^{-i \tau} G\right), \\
& \partial_{t} F^{-}-\frac{p_{x}}{|p|} \partial_{x} F^{-}-E \partial_{p_{x}} F^{-}=-\frac{2|p|}{\varepsilon} \partial_{\tau} F^{-}-\frac{p_{y}}{|p|^{3}} E \Im\left(p e^{-i \tau} G\right), \\
& \partial_{t} G-E \partial_{p_{x}} G=-\frac{2|p|}{\varepsilon} \partial_{\tau} G-i \frac{p_{y}}{|p|^{2}} E G-i \frac{p_{y}}{2|p|^{3}} E \bar{p} e^{i \tau}\left(F^{+}-F^{-}\right) .
\end{aligned}
$$

Because of the presence of an additional variable, this system needs a suitable initial condition $\left(F^{+}(0, x, p, \tau), F^{-}(0, x, p, \tau), G(0, x, p, \tau)\right.$, not only to be well-posed, but also in order to provide a sufficiently smooth solution, that is a solution with time and $(x, p)$ derivatives uniformly bounded with respect to $\varepsilon$, up to some desired order (see [4, 8, 9]). This will be achieved by an appropriate asymptotic expansion (the so-called Chapman-Enskog expansion), which will be also used to derive the asymptotic model.

\section{Chapman-Enskog expansion}

We consider a micro-macro decomposition. To do so, we introduce the average operator $\Pi$ defined for some periodic function $u(\tau)$ on $\mathbb{T}$ as

$$
\Pi u=\frac{1}{2 \pi} \int_{0}^{2 \pi} u(\theta) d \theta
$$

and denote $L u(\tau)=\partial_{\tau} u(\tau)$ (see [4, 8] for more details). The micro-macro decomposition of the unknown $\left(F^{ \pm}, G\right)$ reads

$$
\begin{aligned}
& F^{ \pm}=F_{0}^{ \pm}+F_{1}^{ \pm}, \quad \text { and } G=G_{0}+G_{1}, \\
& \text { with } F_{0}^{ \pm}=\Pi F^{ \pm}, G_{0}=\Pi G, \quad F_{1}^{ \pm}=(I-\Pi) F^{ \pm}, G_{1}=(I-\Pi) G .
\end{aligned}
$$

Inserting these decompositions into (3.17) and applying successively $\Pi$ and $I-\Pi$ leads to a micro-macro model for $\left(F_{0}^{ \pm}, F_{1}^{ \pm}, G_{0}, G_{1}\right)$. An expansion in $\varepsilon$ of the micro 
parts $F_{1}^{ \pm}, G_{1}$ leads to

$$
\begin{aligned}
& G_{1}=-\frac{\varepsilon}{2|p|} L^{-1}\left(i \frac{p_{y}}{2|p|^{3}} E \bar{p} e^{i \tau}\left(F_{0}^{+}-F_{0}^{-}\right)\right)+O\left(\varepsilon^{2}\right), \\
& F_{1}^{+}=\frac{\varepsilon}{2|p|} L^{-1}\left(\frac{p_{y}}{|p|^{3}} E \Im\left(p e^{-i \tau} G_{0}\right)\right)+O\left(\varepsilon^{2}\right), \\
& F_{1}^{-}=-\frac{\varepsilon}{2|p|} L^{-1}\left(\frac{p_{y}}{|p|^{3}} E \Im\left(p e^{-i \tau} G_{0}\right)\right)+O\left(\varepsilon^{2}\right) .
\end{aligned}
$$

Note that $L$ is invertible on functions of zero average with respect to $\tau$ and $L^{-1}\left(e^{ \pm i \tau}\right)=\mp i e^{ \pm i \tau}$. Then we obtain the first order expansion of $F^{ \pm}, G$

$$
\begin{aligned}
& G=G_{0}+G_{1}=G_{0}-\frac{\varepsilon p_{y} \bar{p} E}{4|p|^{4}} e^{i \tau}\left(F_{0}^{+}-F_{0}^{-}\right), \\
& F^{+}=F_{0}^{+}+F_{1}^{+}=F_{0}^{+}+\frac{\varepsilon p_{y} E}{2|p|^{4}} \Im\left(i p e^{-i \tau} G_{0}\right), \\
& F^{-}=F_{0}^{-}+F_{1}^{-}=F_{0}^{-}-\frac{\varepsilon p_{y} E}{2|p|^{4}} \Im\left(i p e^{-i \tau} G_{0}\right) .
\end{aligned}
$$

\section{Derivation of the macro model}

Injecting the decompositions 3.19 in 3.17 and applying $\Pi$ leads to the following macro model

$$
\begin{aligned}
& \partial_{t} F_{0}^{+}+\frac{p_{x}}{|p|} \partial_{x} F_{0}^{+}-E \partial_{p_{x}} F_{0}^{+}=\frac{p_{y}}{|p|^{3}} E \Pi\left(\Im\left(p e^{-i \tau}\left(G_{0}+G_{1}\right)\right)\right), \\
& \partial_{t} F_{0}^{-}-\frac{p_{x}}{|p|} \partial_{x} F_{0}^{-}-E \partial_{p_{x}} F_{0}^{-}=-\frac{p_{y}}{|p|^{3}} E \Pi\left(\Im\left(p e^{-i \tau}\left(G_{0}+G_{1}\right)\right)\right), \\
& \partial_{t} G_{0}-E \partial_{p_{x}} G_{0}=-i \frac{p_{y}}{|p|^{2}} E G_{0}-i \frac{p_{y}}{2|p|^{3}} E \bar{p} \Pi\left(e^{i \tau}\left(F_{0}^{+}+F_{1}^{+}-F_{0}^{-}-F_{1}^{-}\right)\right) .
\end{aligned}
$$

Let us focus on the three right hand side terms including the operator $\Pi$. First, using (3.19), we get for the equations on $F_{0}^{ \pm}$

$$
\Pi\left(\Im\left(p e^{-i \tau}\left(G_{0}+G_{1}\right)\right)\right)=\Pi\left(\Im\left(p e^{-i \tau} G_{1}\right)\right)=-\frac{\varepsilon p_{y} E}{4|p|^{4}} \Im\left(p \bar{p}\left(F_{0}^{+}-F_{0}^{-}\right)\right)=0 .
$$

Then, using again 3.19 , we get for the last term of the equation on $G_{0}$

$\Pi\left(e^{i \tau}\left(F_{0}^{+}+F_{1}^{+}-F_{0}^{-}-F_{1}^{-}\right)\right)=\Pi\left(e^{i \tau}\left(F_{1}^{+}-F_{1}^{-}\right)\right)=\frac{\varepsilon p_{y} E}{|p|^{4}} \Pi\left(e^{i \tau} \Im\left(i p e^{-i \tau} G_{0}\right)\right)$.

From 3.20 and the previous computations, we recover the macro model (3.5).

\section{Derivation of suitable initial data}

We impose the Chapman-Enskog expansion $(3.19)$ at $(t=0, x, p, \tau=0)$ to be consistent with the original initial condition (3.2), that is

$F_{0}^{ \pm}(0, x, p)+F_{1}^{ \pm}(0, x, p, 0)=f_{i n}^{ \pm}(x, p)$ and $G_{0}(0, x, p)+G_{1}(0, x, p, 0)=f_{\text {in }}(x, p)$. 
Using (3.19), we obtain

$$
\begin{aligned}
G_{0}(0, x, p)-\frac{\varepsilon p_{y} \bar{p} E}{4|p|^{4}}\left(F_{0}^{+}(0, x, p)-F_{0}^{-}(0, x, p)\right) & =f_{i n}(x, p), \\
F_{0}^{+}(0, x, p)+\frac{\varepsilon p_{y} E}{2|p|^{4}} \Im\left(i p G_{0}(0, x, p)\right) & =f_{i n}^{+}(x, p), \\
F_{0}^{-}(0, x, p)-\frac{\varepsilon p_{y} E}{2|p|^{4}} \Im\left(i p G_{0}(0, x, p)\right) & =f_{i n}^{-}(x, p) .
\end{aligned}
$$

Considering the difference between the two last equations leads to

$$
F_{0}^{+}(0, x, p)-F_{0}^{-}(0, x, p)=-\frac{\varepsilon p_{y} E}{|p|^{4}} \Im\left(i p G_{0}(0, x, p)\right)+f_{\text {in }}^{+}(x, p)-f_{\text {in }}^{-}(x, p) .
$$

Injecting this in 3.21 to get (after ignoring the $O\left(\varepsilon^{2}\right)$ terms)

$$
\begin{aligned}
f_{i n}(x, p) & =G_{0}(0, x, p)-\frac{\varepsilon p_{y} \bar{p} E}{4|p|^{4}}\left(F_{0}^{+}(0, x, p)-F_{0}^{-}(0, x, p)\right) \\
& =G_{0}(0, x, p)-\frac{\varepsilon p_{y} \bar{p} E}{4|p|^{4}}\left(f_{i n}^{+}(x, p)-f_{i n}^{-}(x, p)\right) .
\end{aligned}
$$

Hence we deduce (3.10). We now consider $\Im\left(i p G_{0}(0, x, p)\right)$

$$
\Im\left(i p G_{0}(0, x, p)\right)=\Im\left(i p f_{i n}(x, p)\right)+\frac{\varepsilon p_{y} E}{4|p|^{2}}\left(f_{i n}^{+}(x, p)-f_{i n}^{-}(x, p)\right),
$$

which injected in $(3.22)$ and 3.23 enables to recover $(3.9)$. To determine the initial condition of the micro model, we simply consider 3.15$)$ at $t=0$ and use the previous computation for $F_{0}^{ \pm}(0, x, p)$ and $G_{0}(x, p)$ to get (3.11) and (3.12).

\section{Derivation of the micro model}

From the Chapman-Enskog expansion (3.19) and relations (3.16), we can write the following micro-macro decomposition of the original unknown

$$
\begin{aligned}
f^{ \pm}(t, x, p) & =F_{0}^{ \pm}(t, x, p) \pm \frac{\varepsilon p_{y} E}{2|p|^{4}} \Im\left(i p e^{-i S / \varepsilon} G_{0}(t, x, p)\right)+h^{ \pm}(t, x, p), \\
g(t, x, p) & =G_{0}(t, x, p)-\frac{\varepsilon p_{y} \bar{p} E}{4|p|^{4}}\left(F_{0}^{+}(t, x, p)-F_{0}^{-}(t, x, p)\right) e^{i S / \varepsilon}+h(t, x, p) .
\end{aligned}
$$

These relations impose in particular that $h^{ \pm}$and $h$ are of order $\varepsilon^{2}$.

To derive an equation for $h^{ \pm}$, we apply the operator $\left(\partial_{t}+\mathcal{T}^{ \pm}\right)$to 3.24 . For $h^{+}$, we get

$$
\left(\partial_{t}+\mathcal{T}^{+}\right) h^{+}=\left(\partial_{t}+\mathcal{T}^{+}\right) f^{+}-\left(\partial_{t}+\mathcal{T}^{+}\right) F_{0}^{+}-\left(\partial_{t}+\mathcal{T}^{+}\right)\left(\frac{\varepsilon p_{y} E}{2|p|^{4}} \Im\left(i p e^{-i S / \varepsilon} G_{0}\right)\right) .
$$

From equation (3.1) on $f^{+}$, we have

$\left(\partial_{t}+\mathcal{T}^{+}\right) f^{+}=\frac{p_{y}}{|p|^{3}} E \Im(p f)=\frac{p_{y}}{|p|^{3}} E \Im\left(p e^{-i S / \varepsilon} g\right)=\frac{p_{y}}{|p|^{3}} E \Im\left(p e^{-i S / \varepsilon}\left(G_{0}+G_{1}+h\right)\right)$, with $G_{1}$ given by (3.19) and where we used $f=e^{-i S / \varepsilon} g$. With the help of the macro equation (3.5) on $F_{0}^{+}$and the above computations, (3.26) then becomes

$$
\left(\partial_{t}+\mathcal{T}^{+}\right) h^{+}=\frac{p_{y}}{|p|^{3}} E \Im\left(p e^{-i S / \varepsilon}\left(G_{0}+G_{1}+h\right)\right)-\left(\partial_{t}+\mathcal{T}^{+}\right)\left(\frac{\varepsilon p_{y} E}{2|p|^{4}} \Im\left(i p e^{-i S / \varepsilon} G_{0}\right)\right) .
$$


Let us compute the first term on the right hand side, which can be decomposed as $R\left[G_{0}\right]+R\left[G_{1}\right]+R[h]$ with $R[\cdot]=\frac{p_{y}}{|p|^{3}} E \Im\left(p e^{-i S / \varepsilon}.\right)$. The term $R\left[G_{1}\right]$ vanishes since, using (3.19)

$$
R\left[G_{1}\right]=\frac{p_{y}}{|p|^{3}} E \Im\left(p e^{-i S / \varepsilon} G_{1}\right)=-\frac{\varepsilon p_{y}^{2} E^{2}}{4|p|^{7}} \Im\left(p e^{-i S / \varepsilon} \bar{p}\left(F_{0}^{+}-F_{0}^{-}\right) e^{i S / \varepsilon}\right)=0 .
$$

Let us consider the last term $\mathcal{C}:=\left(\partial_{t}+\mathcal{T}^{+}\right)\left(\frac{\varepsilon p_{y} E}{2|p|^{4}} \Im\left(i p e^{-i S / \varepsilon} G_{0}\right)\right)$ of 3.27 :

$$
\begin{aligned}
\mathcal{C}= & \mathcal{B}^{+} \Im\left(i p e^{-i S / \varepsilon} G_{0}\right)+\left(\frac{\varepsilon p_{y} E}{2|p|^{4}}\right) \Im\left(\left(\partial_{t}+\mathcal{T}^{+}\right)\left(i p G_{0}\right) e^{-i S / \varepsilon}\right) \\
& +\left(\frac{\varepsilon p_{y} E}{2|p|^{4}}\right) \Im\left(i p G_{0} \frac{-i}{\varepsilon} e^{-i S / \varepsilon}\left(\partial_{t}+\mathcal{T}^{+}\right) S\right),
\end{aligned}
$$

with $\mathcal{B}^{+}=\left(\partial_{t}+\mathcal{T}^{+}\right)\left(\frac{\varepsilon p_{y} E}{2|p|^{4}}\right)$. Since $S$ does not depend on $x, \partial_{t} S+\mathcal{T}^{+} S=2|p|$; then the last term is equal to $-R\left[G_{0}\right]$ and the equation $(3.27)$ on $h^{+}$can be rewritten as

$$
\begin{aligned}
\left(\partial_{t}+\mathcal{T}^{+}\right) h^{+}= & R[h]-\left(\partial_{t}+\mathcal{T}^{+}\right)\left(\frac{\varepsilon p_{y} E}{2|p|^{4}}\right) \Im\left(i p e^{-i S / \varepsilon} G_{0}\right) \\
& -\left(\frac{\varepsilon p_{y} E}{2|p|^{4}}\right) \Im\left(\left(\partial_{t}+\mathcal{T}^{+}\right)\left(i p G_{0}\right) e^{-i S / \varepsilon}\right) .
\end{aligned}
$$

The last term in $(3.29)$ can be written more explicitly using the macro equation (3.5) on $G_{0}$. Indeed, using the notation $\mathcal{D}^{+}:=\Im\left(\left(\partial_{t}+\mathcal{T}^{+}\right)\left(i p G_{0}\right) e^{-i S / \varepsilon}\right)$, we get

$$
\begin{aligned}
\mathcal{D}^{+}= & \Im\left(G_{0} e^{-i S / \varepsilon}\left(\partial_{t}+\mathcal{T}^{+}\right)(i p)\right)+\Im\left(i p e^{-i S / \varepsilon}\left(\partial_{t}+\mathcal{T}^{+}\right) G_{0}\right) \\
= & -\Im\left(G_{0} e^{-i S / \varepsilon} i E\right)+\Im\left(\frac{p p_{y} E G_{0}}{|p|^{2}} e^{-i S / \varepsilon}\right) \\
& +\Im\left(i p e^{-i S / \varepsilon}\left(\frac{p_{x}}{|p|} \partial_{x} G_{0}-i \varepsilon \frac{\bar{p} p_{y}^{2} E^{2}}{2|p|^{7}} \Pi\left(e^{i \tau} \Im\left(i p e^{-i \tau} G_{0}\right)\right)\right)\right) \\
= & -E \Im\left(i G_{0} e^{-i S / \varepsilon}\right)+\frac{p_{y} E}{|p|^{2}} \Im\left(p G_{0} e^{-i S / \varepsilon}\right) \\
& +\frac{p_{x}}{|p|} \Im\left(i p \partial_{x} G_{0} e^{-i S / \varepsilon}\right)+\frac{\varepsilon p_{y}^{2} E^{2}}{4|p|^{5}} \Im\left(p G_{0} e^{-i S / \varepsilon}\right),
\end{aligned}
$$

since $\Pi\left(e^{i \tau} \Im\left(i p e^{-i \tau} G_{0}\right)\right)=p / 2 G_{0}$. Finally, we compute

$$
\left(\partial_{t}+\mathcal{T}^{+}\right)\left(\frac{\varepsilon p_{y} E}{2|p|^{4}}\right)=\left(-E \partial_{p_{x}}\right)\left(\frac{\varepsilon p_{y} E}{2|p|^{4}}\right)=\frac{2 \varepsilon p_{x} p_{y} E^{2}}{|p|^{6}}
$$

which, inserted in $(3.29)$ leads to the following equation on $h^{+}$

$$
\left(\partial_{t}+\mathcal{T}^{+}\right) h^{+}=R[h]-\left(\partial_{t}+\mathcal{T}^{+}\right)\left(\frac{\varepsilon p_{y} E}{2|p|^{4}}\right) \Im\left(i p e^{-i S / \varepsilon} G_{0}\right)-\left(\frac{\varepsilon p_{y} E}{2|p|^{4}}\right) \mathcal{D}^{+},
$$

with $\mathcal{D}^{+}$given by (3.30). This enables to recover the model on $h^{+}$stated in the Proposition.

Similar computations leads to the micro equation on $h^{-}$

$$
\left(\partial_{t}+\mathcal{T}^{-}\right) h^{-}=-R[h]+\left(\partial_{t}+\mathcal{T}^{-}\right)\left(\frac{\varepsilon p_{y} E}{2|p|^{4}}\right) \Im\left(i p e^{-i S / \varepsilon} G_{0}\right)+\left(\frac{\varepsilon p_{y} E}{2|p|^{4}}\right) \mathcal{D}^{-}
$$


where $\mathcal{D}^{-}$is given by

$$
\begin{gathered}
\mathcal{D}^{-}=-\Im\left(G_{0} e^{-i S / \varepsilon} i E\right)+\Im\left(\frac{p p_{y} E G_{0}}{|p|^{2}} e^{-i S / \varepsilon}\right) \\
+\Im\left(i p e^{-i S / \varepsilon}\left(-\frac{p_{x}}{|p|} \partial_{x} G_{0}-i \varepsilon \frac{\bar{p} p_{y}^{2} E^{2}}{2|p|^{7}} \Pi\left(e^{i \tau} \Im\left(i p e^{-i \tau} G_{0}\right)\right)\right)\right) .
\end{gathered}
$$

Then, injecting (3.34) in (3.33) enables to recover the equation on $h^{-}$written in the Proposition.

We now apply $\left(\partial_{t}-E \partial_{p_{x}}\right)$ to 3.15 , to get the following equation on $g$

$$
\begin{aligned}
\left(\partial_{t}-E \partial_{p_{x}}\right) g= & \left(\partial_{t}-E \partial_{p_{x}}\right) G_{0}-\frac{\varepsilon p_{y} E}{4}\left(\partial_{t}-E \partial_{p_{x}}\right)\left(\frac{\bar{p}}{|p|^{4}}\left(F_{0}^{+}-F_{0}^{-}\right) e^{i S / \varepsilon}\right) \\
& +\left(\partial_{t}-E \partial_{p_{x}}\right) h .
\end{aligned}
$$

Using (3.1) and the relation $f=e^{-i S / \varepsilon} g$, the left hand side gives

$$
\begin{aligned}
\left(\partial_{t}-E \partial_{p_{x}}\right) g= & -\frac{i p_{y} E}{|p|^{2}} g-\frac{i p_{y} \bar{p} E}{2|p|^{3}} e^{i S / \varepsilon}\left(f^{+}-f^{-}\right) \\
= & -\frac{i p_{y} E}{|p|^{2}}\left(G_{0}+G_{1}+h\right)-\frac{i p_{y} \bar{p} E}{2|p|^{3}} e^{i S / \varepsilon}\left(F_{0}^{+}+F_{1}^{+}+h^{+}-F_{0}^{-}-F_{1}^{-}-h^{-}\right) \\
= & -\frac{i p_{y} E}{|p|^{2}} G_{0}+\varepsilon \frac{i p_{y}^{2} \bar{p} E^{2}}{4|p|^{6}} e^{i S / \varepsilon}\left(F_{0}^{+}-F_{0}^{-}\right)-\frac{i p_{y} E}{|p|^{2}} h \\
& -\frac{i p_{y} \bar{p} E}{2|p|^{3}} e^{i S / \varepsilon}\left(F_{0}^{+}-F_{0}^{-}\right) \\
& -\frac{i p_{y} \bar{p} E}{2|p|^{3}} e^{i S / \varepsilon}\left(h^{+}-h^{-}\right)-\varepsilon \frac{i p_{y}^{2} \bar{p} E^{2}}{2|p|^{7}} e^{i S / \varepsilon} \Im\left(i p e^{-i S / \varepsilon} G_{0}\right),
\end{aligned}
$$

where we used the expressions 3.19$)$ of $F_{1}^{ \pm}, G_{1}$. The first term on the right hand side of (3.35) gives, using (3.5)

$$
\left(\partial_{t}-E \partial_{p_{x}}\right) G_{0}=-i \varepsilon \frac{\bar{p} p_{y}^{2} E^{2}}{2|p|^{7}} \Pi\left(e^{i \tau} \Im\left(i p e^{-i \tau} G_{0}\right)\right)-\frac{i p_{y} E}{|p|^{2}} G_{0} .
$$

The second term on the right hand side of $(3.35)$ gives

$$
\begin{array}{r}
-\frac{\varepsilon p_{y} E}{4}\left(\partial_{t}-E \partial_{p_{x}}\right)\left(\frac{\bar{p}}{|p|^{4}}\left(F_{0}^{+}-F_{0}^{-}\right) e^{i S / \varepsilon}\right)=-\frac{i p_{y} \bar{p} E}{2|p|^{3}}\left(F_{0}^{+}-F_{0}^{-}\right) e^{i S / \varepsilon} \\
+\frac{\varepsilon p_{y} E^{2}}{4} \partial_{p_{x}}\left(\frac{\bar{p}}{|p|^{4}}\right)\left(F_{0}^{+}-F_{0}^{-}\right) e^{i S / \varepsilon}-\frac{\varepsilon p_{y} p_{x} \bar{p} E}{4|p|^{5}} \partial_{x}\left(F_{0}^{+}+F_{0}^{-}\right) e^{i S / \varepsilon} .
\end{array}
$$

Then, we obtain from the previous computations injected in 3.35

$$
\begin{aligned}
\left(\partial_{t}-E \partial_{p_{x}}\right) h= & \varepsilon \frac{i p_{y}^{2} \bar{p} E^{2}}{4|p|^{6}} e^{i S / \varepsilon}\left(F_{0}^{+}-F_{0}^{-}\right)-\frac{i p_{y} E}{|p|^{2}} h \\
& -\frac{i p_{y} \bar{p} E}{2|p|^{3}} e^{i S / \varepsilon}\left(h^{+}-h^{-}\right)-\varepsilon \frac{i p_{y}^{2} \bar{p} E^{2}}{2|p|^{7}} e^{i S / \varepsilon} \Im\left(i p e^{-i S / \varepsilon} G_{0}\right) \\
& +i \varepsilon \frac{\bar{p} p_{y}^{2} E^{2}}{2|p|^{7}} \Pi\left(e^{i \tau} \Im\left(i p e^{-i \tau} G_{0}\right)\right) \\
& -\frac{\varepsilon p_{y} E^{2}}{4} \partial_{p_{x}}\left(\frac{\bar{p}}{|p|^{4}}\right)\left(F_{0}^{+}-F_{0}^{-}\right) e^{i S / \varepsilon}-\frac{\varepsilon p_{y} p_{x} \bar{p} E}{4|p|^{5}} \partial_{x}\left(F_{0}^{+}+F_{0}^{-}\right) e^{i S / \varepsilon} .
\end{aligned}
$$


We now focus on the two terms (which we call $\mathcal{A}_{1}$ and $\mathcal{A}_{2}$ ) which involve $G_{0}$. Their sum gives

$$
\begin{aligned}
\mathcal{A}_{1}+\mathcal{A}_{2} & =-\varepsilon \frac{i p_{y}^{2} \bar{p} E^{2}}{2|p|^{7}}\left[e^{i S / \varepsilon} \Im\left(i p e^{-i S / \varepsilon} G_{0}\right)-\Pi\left(e^{i \tau} \Im\left(i p e^{-i \tau} G_{0}\right)\right)\right] \\
& =-\varepsilon \frac{i p_{y}^{2} \bar{p} E^{2}}{2|p|^{7}}\left[\frac{1}{2} p G_{0}+\frac{1}{2} \bar{p} e^{2 i S / \varepsilon} \bar{G}_{0}-\frac{1}{2} p G_{0}\right] \\
& =-\varepsilon \frac{i p_{y}^{2} \bar{p}^{2} E^{2}}{4|p|^{7}} e^{2 i S / \varepsilon} \bar{G}_{0} .
\end{aligned}
$$

Finally, after some simplifications, we get the equation for $h$ written in the Proposition.

Note that in this context of homogeneous field $E$, we can solve explicitly the equation on the phase $S$ (the non homogeneous case is treated in Appendix A). Indeed, from (3.3), one gets, using the method of the characteristics and integrating on $[0, t]$

$$
\begin{aligned}
S\left(t, x, p_{x}-E t, p_{y}\right)-S\left(0, x, p_{x}, p_{y}\right) & =2 \int_{0}^{t} \sqrt{\left(p_{x}-E s\right)^{2}+p_{y}^{2}} d s \\
& =2 \frac{p_{y}^{2}}{E} \int_{-p_{x} / p_{y}}^{\left(E t-p_{x}\right) / p_{y}} \sqrt{1+u^{2}} d u .
\end{aligned}
$$

From the relation $\int_{0}^{u} \sqrt{1+v^{2}} d v=\frac{1}{2} \xi(u)$ with $\xi(u)=u \sqrt{1+u^{2}}+\ln \left(u+\sqrt{1+u^{2}}\right)$, we finally obtain

$$
S\left(t, x, p_{x}, p_{y}\right)=\frac{p_{y}^{2}}{E}\left[-\xi\left(\frac{p_{x}}{p_{y}}\right)+\xi\left(\frac{p_{x}+E t}{p_{y}}\right)\right] .
$$

In the non homogeneous case, the phase $S$ does not depend on $x$.

\section{Uniform estimates of the micro unknown derivatives}

In this section, we prove some uniform smoothness of the solution of the model stated in Proposition 3.1. More precisely, we show that the derivatives (in $t, x, p_{x}$ ) of the micro unknown $h^{ \pm}, h$ are uniformly bounded with respect to $\varepsilon$. It is clear that the macro unknown $\left(F_{0}^{ \pm}, G_{0}\right)$ and the phase $S$ are smooth since their equations do not contain any stiff terms in $\varepsilon$.

First we rewrite in an abstract way the micro equations (3.7) as

$\partial_{t} H+\mathcal{T} H=\left(A e^{i S / \varepsilon}+\bar{A} e^{-i S / \varepsilon}\right) H+\varepsilon\left(\alpha e^{i S / \varepsilon}+\bar{\alpha} e^{-i S / \varepsilon}\right)+\varepsilon\left(\beta e^{2 i S / \varepsilon}+\bar{\beta} e^{-2 i S / \varepsilon}\right)+\gamma H$,

where $H(t, x, p)=\left(h^{+}, h^{-}, \Re(h), \Im(h)\right)(t, x, p), A(t, x, p), \gamma(t, x, p)$ are complex $4 \times 4$ matrices, $\alpha(t, x, p), \beta(t, x, p) \in \mathbb{C}^{4}$ and $S(t, p) \in \mathbb{R}$ is the phase solution of (3.3). Moreover, we define $\mathcal{T} H$ as

$$
\mathcal{T} H=\left(\begin{array}{l}
\frac{p_{x}}{p \mid} \partial_{x} h^{+}-E \partial_{p_{x}} h^{+} \\
\frac{p_{x}}{|p|} \partial_{x} h^{-}-E \partial_{p_{x}} h^{-} \\
-E \partial_{p_{x}} \Re(h) \\
-E \partial_{p_{x}} \Im(h)
\end{array}\right) .
$$


Note that $A, \alpha, \beta, \gamma$ depend on the solution of the macro equations (3.5) (as such, they depend on $\varepsilon$ ), which are uniformly bounded with respect to $\varepsilon$ since there is no stiffness in the macro equations. We will assume in the sequel that $A, \alpha, \beta, \gamma$ and their derivatives (up to the second order) are bounded.

The initial condition $H\left(0, x, p_{x}\right)$ is given by (3.11) and (3.12) and, assuming the initial conditions $\left(f_{i n}^{ \pm}, f_{i n}\right)$ bounded and $\mathcal{C}^{2}$ with respect to $x, p_{x}$, we will simply use in the following that $H\left(0, x, p_{x}\right)=O\left(\varepsilon^{2}\right)$ in the $L_{x, p_{x}}^{\infty}$ norm.

Theorem 4.1. Let $H$ be the solution of (4.1) on $\left[0, T_{0}\right]$ with initial data $H(0)$ satisfying $\|H(0)\|_{L_{x, p_{x}}^{\infty}}=O\left(\varepsilon^{2}\right)$. Let $A, \alpha, \beta, \gamma$ be $\mathcal{C}^{2}$ functions with respect to $t, x, p_{x}$ which are $W^{2, \infty}$ with respect to $x, p_{x}$. We assume in addition that $p_{y} \neq 0$. Then, we have the following estimates for $H$ : for all $t \in\left[0, T_{0}\right]$,

$$
\begin{gathered}
\|H(t)\|_{L_{x, p_{x}}^{\infty}} \leq C \varepsilon^{2}, \quad\left\|\partial_{t} H(t)\right\|_{L_{x, p_{x}}^{\infty}} \leq C \varepsilon, \text { and }\left\|\partial_{t}^{2} H(t)\right\|_{L_{x, p_{x}}^{\infty}} \leq C, \\
\left\|\partial_{p_{x}} H(t)\right\|_{L_{x, p_{x}}^{\infty}} \leq C \varepsilon, \text { and }\left\|\partial_{p_{x}}^{2} H(t)\right\|_{L_{x, p_{x}}^{\infty} \leq C,} \leq C, \\
\left\|\partial_{x}^{\ell} H(t)\right\|_{L_{x, p_{x}}^{\infty}} \leq C_{\ell} \varepsilon^{2}, \text { for } \ell=1,2, \ldots,
\end{gathered}
$$

where the constants $C>0, C_{\ell}>0$ are independent of $\varepsilon$ and $t$.

Proof. As in [6], we consider the following change of unknown

$$
W(S(t, p), x, p)=H(t, x, p),
$$

where $S$ satisfies (3.3). Observe that, for all $p_{x} \in \mathbb{R}$, the phase $S\left(t,\left(p_{x}, p_{y}\right)\right)$ is an increasing function in $t$ (recall that $E$ does not depend on $x$, so $S$ does not either). This means that the map $t \mapsto s=S\left(t,\left(p_{x}, p_{y}\right)\right)$ can be seen as a change of variable in time. Then $W(s, x, p)$ satisfies

$$
\begin{aligned}
\partial_{s} W+\frac{1}{2|p|} \mathcal{T} W= & \frac{1}{2|p|}\left(A e^{i s / \varepsilon}+\bar{A} e^{-i s / \varepsilon}\right) W+\frac{\varepsilon}{2|p|}\left(\alpha e^{i s / \varepsilon}+\bar{\alpha} e^{-i s / \varepsilon}\right) \\
& +\frac{\varepsilon}{2|p|}\left(\beta e^{2 i s / \varepsilon}+\bar{\beta} e^{-2 i s / \varepsilon}\right)+\frac{\gamma}{2|p|} W,
\end{aligned}
$$

with the initial condition $W(0, x, p)=O\left(\varepsilon^{2}\right)$ since $S(0, p)=0$ and $H(0, x, p)=$ $O\left(\varepsilon^{2}\right)$. In the following, we will use a standard lemma to prove that the derivatives of $W$ with respect to $s, x, p_{x}$, solution of (4.3), are uniformly bounded in the space $L_{x, p_{x}}^{\infty}$ of bounded functions of $x$ and $p_{x}$.

Lemma 4.2. Consider the following partial differential equation

$$
\begin{gathered}
\partial_{s} y^{\varepsilon}+\mathcal{A} \cdot \nabla_{x, p} y^{\varepsilon}=\left(A e^{i s / \varepsilon}+\bar{A} e^{-i s / \varepsilon}\right) y^{\varepsilon}+\left(\alpha e^{i s / \varepsilon}+\bar{\alpha} e^{-i s / \varepsilon}\right) \\
+\left(\beta e^{2 i s / \varepsilon}+\bar{\beta} e^{-2 i s / \varepsilon}\right)+\varepsilon \xi+\gamma y^{\varepsilon}, \\
\forall s, x, p \in[0, T] \times \mathbb{R} \times \mathbb{R}, y^{\varepsilon}(0, x, p)=y_{0}^{\varepsilon}(x, p),
\end{gathered}
$$

with $y^{\varepsilon}, \xi$ (resp. $\alpha$ and $\beta$ ) : $[0, T] \times \mathbb{R} \times \mathbb{R} \mapsto \mathbb{R}^{4}$ (resp. $\mathbb{C}^{4}$ ), the vector field $\mathcal{A}=\left(\mathcal{A}_{1}, \ldots, \mathcal{A}_{4}\right)$ is such that $\mathcal{A}_{k}: \mathbb{R}^{2} \mapsto \mathbb{R}^{2}, k=1, \ldots, 4$ are Lipschitz functions in $L_{x, p}^{\infty}$, and $A, \gamma$ are $4 \times 4$ matrices whose coefficients are complex valued functions of $(s, x, p)$. Assume that there exists a constant $C>0$ independent of $\varepsilon$ such

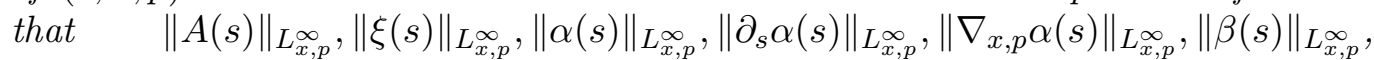
$\left\|\partial_{s} \beta(s)\right\|_{L_{x, p}^{\infty}},\left\|\nabla_{x, p} \beta(s)\right\|_{L_{x, p}^{\infty}},\|\gamma(s)\|_{L_{x, p}^{\infty}} \leq C, \forall s \in[0, T]$. Then, we have $\left\|y^{\varepsilon}(s)\right\|_{L_{x, p}^{\infty}} \leq C_{T}\left(\varepsilon+\left\|y_{0}^{\varepsilon}\right\|_{L_{x, p}^{\infty}}\right), \forall s \in[0, T]$, where the constant $C_{T}>0$ depends on $T$ but is independent of $\varepsilon$. 
Proof of Lemma 4.2. To prove the Lemma, we first define the characteristics equation by introducing $Z(s)=(x(s), p(s)) \in \mathbb{R}^{2}$

$$
\dot{Z}(s)=\mathcal{A}_{k}(Z(s)), \quad Z(0)=(x, p),
$$

whose unique solution is written as $Z(s ; 0, x, p)$ (thanks to the Lipschitz character of $\left.\mathcal{A}_{k}\right)$. Then, one can write the $k$-th component of (4.4) as

$$
\begin{aligned}
\partial_{s}\left(y_{k}^{\varepsilon}(s, Z(s ; 0, x, p))\right)= & {\left[\left(A e^{i s / \varepsilon}+\bar{A} e^{-i s / \varepsilon}\right) y^{\varepsilon}+\left(\alpha e^{i s / \varepsilon}+\bar{\alpha} e^{-i s / \varepsilon}\right)\right.} \\
& \left.+\left(\beta e^{2 i s / \varepsilon}+\bar{\beta} e^{-2 i s / \varepsilon}\right)+\varepsilon \xi+\gamma y^{\varepsilon}\right]_{k}(s, Z(s ; 0, x, p)) .
\end{aligned}
$$

Then, denoting by $\tilde{y}_{k}(s, z):=y_{k}^{\varepsilon}(s, Z(s ; 0, x, p))$ leads to (using the same notations for $A, \alpha, \beta$ and $\gamma$ )

$$
\partial_{s} \tilde{y}=\left(\tilde{A} e^{i s / \varepsilon}+\tilde{\bar{A}} e^{-i s / \varepsilon}\right) \tilde{y}+\left(\tilde{\alpha} e^{i s / \varepsilon}+\tilde{\bar{\alpha}} e^{-i s / \varepsilon}\right)+\left(\tilde{\beta} e^{2 i s / \varepsilon}+\tilde{\bar{\beta}} e^{-2 i s / \varepsilon}\right)+\varepsilon \tilde{\xi}+\tilde{\gamma} \tilde{y} .
$$

We now integrate in time between 0 and $s$ to get the following estimate (using assumptions on $A, \alpha, \beta, \xi$ and $\gamma$ )

$$
\|\tilde{y}(s)\|_{L_{z}^{\infty}} \leq\|y(0)\|_{L_{z}^{\infty}}+C \int_{0}^{s}\|\tilde{y}(\sigma)\|_{L_{z}^{\infty}} d \sigma+C \varepsilon .
$$

To get the last term, we performed integrations by parts for the source terms, for instance

$$
\int_{0}^{s} \tilde{\alpha}(\sigma, z) e^{i \sigma / \varepsilon} d \sigma=-i \varepsilon\left[\tilde{\alpha}(s, z) e^{i s / \varepsilon}-\tilde{\alpha}(0, z)\right]+i \varepsilon \int_{0}^{s} \partial_{s} \tilde{\alpha}(\sigma, z) e^{i \sigma / \varepsilon} d \sigma .
$$

From $\partial_{s} \tilde{\alpha}(\sigma, z)=\left[\partial_{s} \alpha+\left(\nabla_{x, p} \alpha\right) \mathcal{A}_{k}\right](s, Z(s ; 0, x, p))$ and the assumptions on $\alpha$, we ensure that the last term is uniformly bounded. Using now Gronwall's lemma enables to prove $\|\tilde{y}(s)\|_{L_{z}^{\infty}} \leq C_{T}\left(\varepsilon+\|y(0)\|_{L_{x, p}^{\infty}}\right)$ so that $\left\|y^{\varepsilon}(s)\right\|_{L_{x, p}^{\infty}} \leq C_{T}(\varepsilon+$ $\left.\left\|y_{0}^{\varepsilon}\right\|_{L_{x, p}^{\infty}}\right), \forall s \in[0, T]$.

Thanks to this lemma, we will successively prove that the derivatives in $s, x, p_{x}$ of $W$, the solution of 4.3), are uniformly bounded.

Estimate of $W / \varepsilon^{2}$.

Dividing (4.3) by $\varepsilon$ leads to the following system satisfied by $\check{W}=W / \varepsilon$ (still using the same notations)

$$
\begin{aligned}
\partial_{s} \check{W}+\frac{1}{2|p|} \mathcal{T} \check{W}= & \frac{1}{2|p|}\left(A e^{i s / \varepsilon}+\bar{A} e^{-i s / \varepsilon}\right) \check{W}+\frac{1}{2|p|}\left(\alpha e^{i s / \varepsilon}+\bar{\alpha} e^{-i s / \varepsilon}\right) \\
& +\frac{1}{2|p|}\left(\beta e^{2 i s / \varepsilon}+\bar{\beta} e^{-2 i s / \varepsilon}\right)+\frac{\gamma}{2|p|} \check{W}
\end{aligned}
$$

with the initial condition $\check{W}(0, x, p)=O(\varepsilon)$. By using Lemma 4.2 , we get

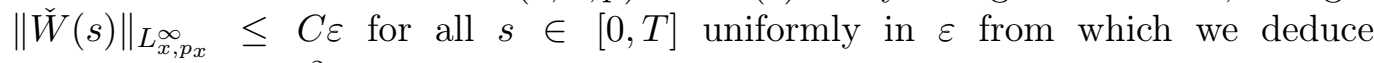

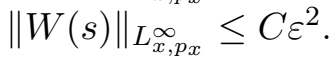


Estimate of $\left(\partial_{x} W\right) / \varepsilon^{2}$.

Using the same strategy as before, i.e. differentiating (4.3) with respect to $x$ and dividing by $\varepsilon$ leads to the following system satisfied by $W=\left(\partial_{x} W\right) / \varepsilon$

$$
\begin{aligned}
\partial_{s} \check{W}+\frac{1}{2|p|} \mathcal{T} \check{W}= & \frac{1}{2|p|}\left(\partial_{x} A e^{i s / \varepsilon}+\partial_{x} \bar{A} e^{-i s / \varepsilon}\right) \frac{W}{\varepsilon}+\frac{1}{2|p|}\left(A e^{i s / \varepsilon}+\bar{A} e^{-i s / \varepsilon}\right) \check{W} \\
& +\frac{1}{2|p|}\left(\partial_{x} \alpha e^{i s / \varepsilon}+\partial_{x} \bar{\alpha} e^{-i s / \varepsilon}\right)+\frac{1}{2|p|}\left(\partial_{x} \beta e^{2 i s / \varepsilon}+\partial_{x} \bar{\beta} e^{-2 i s / \varepsilon}\right) \\
& +\frac{\partial_{x} \gamma}{2|p|} \frac{W}{\varepsilon}+\frac{\gamma}{2|p|} \check{W},
\end{aligned}
$$

with the initial condition $\check{W}(0, x, p)=O(\varepsilon)$. Then, thanks to the previous uniform estimate on $W / \varepsilon^{2}$, we can use Lemma 4.2 to conclude that $\|\check{W}(s)\|_{L_{x, p_{x}}^{\infty} \leq C \varepsilon \text { so }}$ that $\left\|\left(\partial_{x} W(s)\right) / \varepsilon^{2}\right\|_{L_{x, p_{x}}^{\infty}} \leq C$ uniformly in $\varepsilon$. Note that the same strategy ensures the uniform boundedness of $\left(\partial_{x}^{\ell} W\right) / \varepsilon^{2}, \quad \ell=2,3, \ldots$, since the derivation with respect to $x$ does not generate $1 / \varepsilon$ terms in the right hand side.

Estimate of $\left(\partial_{p_{x}} W\right) / \varepsilon^{2}$.

Using the same strategy as before, i.e. deriving (4.3) with respect to $p_{x}$ and dividing by $\varepsilon$ leads to the following system satisfied by $W=\left(\partial_{p_{x}} W\right) / \varepsilon$

$$
\begin{aligned}
\partial_{s} \check{W}+\frac{1}{2|p|} \mathcal{T} \check{W} & =\left(\partial_{p_{x}}\left(\frac{1}{2|p|} A\right) e^{i s / \varepsilon}+\partial_{p_{x}}\left(\frac{1}{2|p|} \bar{A}\right) e^{-i s / \varepsilon}\right) \frac{W}{\varepsilon} \\
+ & \frac{1}{2|p|}\left(A e^{i s / \varepsilon}+\bar{A} e^{-i s / \varepsilon}\right) \check{W}+\left(\partial_{p_{x}}\left(\frac{1}{2|p|} \alpha\right) e^{i s / \varepsilon}+\partial_{p_{x}}\left(\frac{1}{2|p|} \bar{\alpha}\right) e^{-i s / \varepsilon}\right) \\
+ & \left(\partial_{p_{x}}\left(\frac{1}{2|p|} \beta\right) e^{2 i s / \varepsilon}+\partial_{p_{x}}\left(\frac{1}{2|p|} \bar{\beta}\right) e^{-2 i s / \varepsilon}\right) \\
+ & \partial_{p_{x}}\left(\frac{\gamma}{2|p|}\right) \frac{W}{\varepsilon}+\frac{\gamma}{2|p|} \check{W}-\frac{1}{\varepsilon} \partial_{p_{x}}\left(\frac{p}{|p|}\right) \mathcal{J} \partial_{x} W
\end{aligned}
$$

with the initial condition $\breve{W}(0, s, p)=O(\varepsilon)$ and where the matrix $\mathcal{J}$ is given by

$$
\mathcal{J}=\left(\begin{array}{llll}
1 & 0 & 0 & 0 \\
0 & -1 & 0 & 0 \\
0 & 0 & 0 & 0 \\
0 & 0 & 0 & 0
\end{array}\right)
$$

Let us remark that the last term in (4.6) comes from the transport part $p /|p| \partial_{x}$ which, after differentiation in $p_{x}$, generates a source term $\frac{1}{\varepsilon} \partial_{p_{x}}\left(\frac{p}{|p|}\right) \mathcal{J} \partial_{x} W$ and a transport term for the equation on $\check{W}$. Such a source term did not appear in the previous equation (4.5) on $\left(\partial_{x} W\right) / \varepsilon$ since in this study $E$ does not depend on $x$ (let us remark that in this step, the case of a space dependent $E$ can also be dealt with). Then, thanks to the above estimates on $W / \varepsilon^{2}, \partial_{x} W / \varepsilon^{2}$, we can use again Lemma 4.2 to conclude that $\|\breve{W}(s)\|_{L_{x, p_{x}}^{\infty}} \leq C \varepsilon$ so that $\left\|\left(\partial_{p_{x}} W(s)\right) / \varepsilon^{2}\right\|_{L_{x, p_{x}}^{\infty}} \leq C$ uniformly in $\varepsilon$. The same strategy ensures the uniform boundedness of $\left.\left(\partial_{p_{x}}^{\ell} W\right) / \varepsilon^{2}\right)$ $\ell=2,3, \ldots$. 
Estimate of $\partial_{s} W / \varepsilon$.

Considering (4.3) divided by $\varepsilon$, and using the above estimates, we directly obtain $\left\|\left(\partial_{s} W(s)\right) / \varepsilon\right\|_{L_{x, p_{x}}^{\infty}} \leq C$.

Estimate of $\left(\partial_{x p_{x}}^{2} W\right) / \varepsilon^{2}$.

Differentiating (4.6) with respect to $x$ and dividing by $\varepsilon$ leads to the following equation satisfied by $\check{W}=\left(\partial_{x p_{x}}^{2} W\right) / \varepsilon$

$$
\partial_{s} \check{W}+\frac{1}{2|p|} \mathcal{T} \check{W}=\mathrm{rhs},
$$

with $\check{W}(0)=O\left(\varepsilon^{2}\right)$ and where rhs depends on the derivatives in $x, p_{x}$ of $A, \alpha, \beta, \gamma$ and on $\partial_{x} W / \varepsilon, \check{W}, \partial_{x}^{2} W / \varepsilon$. Then, we can use Lemma 4.2 and the above estimates to get $\left\|\partial_{x, p_{x}}^{2} W(s) / \varepsilon^{2}\right\|_{L_{x, p_{x}}^{\infty}} \leq C$.

Estimate of $\left(\partial_{s, x}^{2} W\right) / \varepsilon\left(\right.$ resp. $\left.\left(\partial_{s, p_{x}}^{2} W\right) / \varepsilon\right)$.

From (4.5) (resp. (4.6) ) and from the estimates on $\partial_{x} W / \varepsilon, \partial_{p_{x}} W / \varepsilon^{2}, W / \varepsilon^{2}$, we

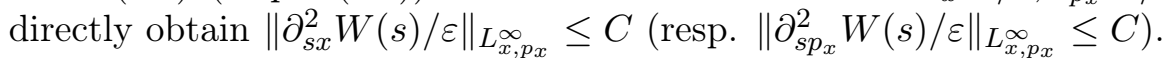

Estimate of $\partial_{s}^{2} W$.

Differentiating (4.3) with respect to $s$ leads to the following system

$$
\begin{aligned}
\partial_{s}^{2} W+\frac{1}{2|p|} \mathcal{T} \partial_{s} W= & \frac{1}{2|p|}\left(\partial_{s} A e^{i s / \varepsilon}+A \frac{i e^{i s / \varepsilon}}{\varepsilon}+\partial_{s} \bar{A} e^{-i s / \varepsilon}-\bar{A} \frac{i e^{-i s / \varepsilon}}{\varepsilon}\right) W \\
& +\left(A e^{i s / \varepsilon}+\bar{A} e^{-i s / \varepsilon}\right) \partial_{s} W \\
& +\frac{1}{2|p|}\left(\varepsilon \partial_{s} \alpha e^{i s / \varepsilon}+\alpha i e^{i s / \varepsilon}+\varepsilon \bar{\alpha} e^{-i s / \varepsilon}-\alpha i e^{-i s / \varepsilon}\right) \\
& +\frac{1}{2|p|}\left(\varepsilon \partial_{s} \beta e^{2 i s / \varepsilon}+2 i \beta e^{2 i s / \varepsilon}+\varepsilon \partial_{s} \bar{\beta} e^{-2 i s / \varepsilon}-2 i \bar{\beta} e^{-2 i s / \varepsilon}\right) \\
& +\frac{\partial_{s} \gamma}{2|p|} W+\frac{\gamma}{2|p|} \partial_{s} W
\end{aligned}
$$

with $W(0, s, p)=O\left(\varepsilon^{2}\right)$ and $\partial_{s} W(0, s, p)=O(\varepsilon)$. Thanks to the previous estimates, we have $\partial_{s x}^{2} W, \partial_{s p_{x}}^{2} W, W / \varepsilon, \partial_{s} W, W$ uniformly bounded. Then, we deduce directly from (4.8) that $\partial_{s}^{2} W$ is uniformly bounded. Let us remark that if we differentiate (4.8) with respect to $s$ again, some stiff term in $1 / \varepsilon$ will appear in the third and fourth terms of the right hand side.

\section{Conclusion.}

We then use the following relations between $W$ and $H$

$$
\begin{aligned}
\partial_{t} H(t, x, p) & =\partial_{t} S(t, p) \partial_{s} W(S(t, p), x, p), \\
\partial_{t}^{2} H & =\left(\partial_{t} S\right)^{2} \partial_{s}^{2} W+\partial_{t}^{2} S \partial_{s} W \\
\partial_{x}^{\ell} H & =\partial_{x}^{\ell} W, \quad \ell=1,2, \ldots, \\
\partial_{p_{x}} H & =\partial_{p_{x}} S \partial_{s} W+\partial_{p_{x}} W \\
\partial_{p_{x}}^{2} H & =\left(\partial_{p_{x}} S\right)^{2} \partial_{s}^{2} W+\partial_{p_{x}}^{2} S \partial_{s} W+\partial_{p_{x}} S \partial_{s p_{x}}^{2} W+\partial_{p_{x}}^{2} W,
\end{aligned}
$$


where $S(t, p)$, given by (3.3) with $E$ independent from $x$, has its derivatives in $t$ and $p_{x}$ uniformly bounded. The above estimates and the relations between $H$ and $W$ conclude the proof.

\section{Numerical scheme}

\subsection{A model equation and the proof of uniform numerical accuracy in time.}

For simplicity, our numerical results are restricted to the case of an homogeneous field $E$. To present our numerical scheme, we shall concentrate on a model equation of the form

$$
\partial_{t} u+\frac{p_{x}}{|p|} \partial_{x} u-E \partial_{p_{x}} u=e^{i S\left(t, p_{x}\right) / \varepsilon}\left(\varepsilon \alpha\left(t, x, p_{x}\right)+\beta\left(t, x, p_{x}\right) u\right),
$$

with initial condition $u(0)=u_{0}=O\left(\varepsilon^{2}\right)$ (see (3.11)-(3.12), and assume the initial conditions $\left(f_{i n}^{ \pm}, f_{i n}\right)$ bounded and $\mathcal{C}^{2}$ with respect to $\left.x, p_{x}\right)$, and with coefficients $\alpha, \beta \in \mathcal{C}^{\infty}$ with respect to $t, x, p_{x}$ such that, $\forall 0 \leq t \leq T_{0}$

$$
\left\|\partial_{t}^{\ell} \partial_{x}^{m} \partial_{p_{x}}^{k} \alpha(t)\right\|_{L_{x, p_{x}}^{\infty}}+\left\|\partial_{t}^{\ell} \partial_{x}^{m} \partial_{p_{x}}^{k} \beta(t)\right\|_{L_{x, p_{x}}^{\infty}} \leq C, \quad \text { for all } k, \ell, m \in \mathbb{N},
$$

with $C$ independent of $\varepsilon$ but may depend on $k, \ell, m$. Then, using Theorem 4.1 , the solution $u$ of (5.1) satisfies the following estimates uniform in $\varepsilon$,

$$
\sum_{k=0}^{2} \frac{1}{\varepsilon^{2-k}}\left(\left\|\partial_{t}^{k} u(t)\right\|_{L_{x, p_{x}}^{\infty}}+\left\|\partial_{p_{x}}^{k} u(t)\right\|_{L_{x, p_{x}}^{\infty}}\right)+\frac{1}{\varepsilon^{2}}\left\|\partial_{x}^{\ell} u(t)\right\|_{L_{x, p_{x}}^{\infty}} \leq C, \quad \text { for } \ell=0,1, \ldots
$$

This scheme can be easily adapted to the macro model (3.5), which contains no stiff term, and to the micro equations (3.7) which are in a form close to (5.1).

We shall only describe a semi-discrete scheme in time. The $x$ and $p_{x}$ variables are further discretized on a domain with periodic boundary conditions. The fast discrete Fourier transform is used to deal with advection term in the spatial variable $x$, which ensures spectral accuracy in this direction (thanks to the previous estimates on $\left.\partial_{x}^{\ell} u\right)$. However, in the $p_{x}$ variable, we do not have enough smoothness. Then, we integrate exactly the transport in $p_{x}$, using characteristic variables, by introducing the unknown $v\left(t, x, p_{x}\right)=u\left(t, x, p_{x}-E t\right)$ which solves the equation

$$
\partial_{t} v+\frac{p_{x}-E t}{\left|\left(p_{x}-E t, p_{y}\right)\right|} \partial_{x} v=e^{i \widetilde{S}\left(t, p_{x}\right) / \varepsilon}\left(\varepsilon \widetilde{\alpha}\left(t, x, p_{x}\right)+\widetilde{\beta}\left(t, x, p_{x}\right) v\right),
$$

where we have denoted

$\widetilde{S}\left(t, p_{x}\right)=S\left(t, p_{x}-E t\right), \quad \widetilde{\alpha}\left(t, x, p_{x}\right)=\alpha\left(t, x, p_{x}-E t\right), \quad \beta\left(t, x, p_{x}\right)=\beta\left(t, x, p_{x}-E t\right)$.

Then, $p_{x}$ is a parameter in (5.4) and the scheme will be exact with respect to this direction. However, when one wants to reconstruct the original solution $u\left(t, x, p_{x}\right)=$ $v\left(t, x, p_{x}+E t\right)$ at the final time $t$ and on a mesh in $p_{x}$, one needs to interpolate $v$ at $p_{x}+E t$. This can be done using trigonometric interpolation which leads to second order uniform accuracy in $p_{x}$ since only $\partial_{p_{x}}^{\ell} u$ for $\ell \leq 2$ are uniformly bounded. To avoid this restriction, we propose to use a staggered mesh in $p_{x}$ on which $u$ is computed so that the interpolation is exact and does not introduce error. To do so, we first truncate the $p_{x}$ domain by considering the interval $\left[-v_{\max }, v_{\max }\right]$ with 
$v_{\max }>0$ and we define the following mesh (on which the solution $v$ of $(5.4)$ is computed): $p_{j}=-v_{\max }+j \Delta p$ for $j=0, \ldots, N_{p}\left(N_{p} \in \mathbb{N}^{\star}\right)$ with $\Delta p=\left(2 v_{\max }\right) / N_{p}$ the mesh size. Then, we introduce $\iota \in\left[0,1\left[\right.\right.$ as $\iota=\frac{E t}{\Delta p}-\left\lfloor\frac{E t}{\Delta p}\right\rfloor$, and consider the following staggered mesh in $p_{x}$ (on which the function $u$ solution of (5.1) will be computed $): q_{\ell}=-v_{\max }+(\ell-\iota) \Delta p\left(\ell=0, \ldots, N_{p}\right)$. Assuming periodic boundary conditions, this ensures that the original solution $u$ is computed exactly on the staggered mesh.

Let $\Delta t>0$ and $t^{n}=n \Delta t \leq T_{0}$. We split (5.4) into two parts and use the Strang splitting scheme. To analyze the splitting we rewrite (5.4) as

$$
\partial_{t} v+a(t, p) \partial_{x} v=e^{i \widetilde{S}\left(t, p_{x}\right) / \varepsilon}\left(\varepsilon \widetilde{\alpha}\left(t, x, p_{x}\right)+\widetilde{\beta}\left(t, x, p_{x}\right) v\right),
$$

with $a(t, p)=\frac{p_{x}-E t}{\left|\left(p_{x}-E t, p_{y}\right)\right|}$. To overcome the non autonomous character, we introduce an additional variable $w(t)=t$ so that we consider the following system satisfied by $y=(v, w)$

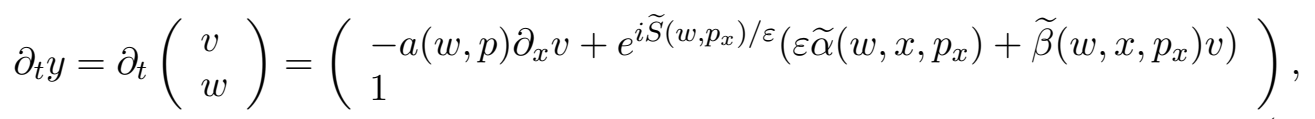

with the initial condition: $v(0)=u_{0}, w(0)=0$. Then, the splitting we consider reads

$$
\partial_{t}\left(\begin{array}{l}
v \\
w
\end{array}\right)=\left(\begin{array}{l}
-a(w, p) \partial_{x} v \\
0
\end{array}\right)
$$

and

$$
\partial_{t}\left(\begin{array}{l}
v \\
w
\end{array}\right)=\left(\begin{array}{l}
e^{i \widetilde{S}\left(w, p_{x}\right) / \varepsilon}\left(\varepsilon \widetilde{\alpha}\left(w, x, p_{x}\right)+\widetilde{\beta}\left(w, x, p_{x}\right) v\right) \\
1
\end{array}\right)
$$

To reach second order accuracy in time, a Strang splitting is used. Let us detail in the following the steps to pass from the initial condition $y^{n}=\left(v^{n}, w^{n}\right)$ at time $t^{n}$ to $y^{n+1}=\left(v^{n+1}, w^{n+1}\right)$ at time $t^{n+1}$ :

(i) First, on $\left[t^{n}, t^{n+1 / 2}\right]$, we solve

$$
\partial_{t} v=-a(w, p) \partial_{x} v=0, \quad \partial_{t} w=0,
$$

with initial data $\left(v^{n}, t^{n}\right)$, which has the following explicit solution since during this step, $w(t)=t^{n}$ so that $a(w, p)=a\left(t^{n}, p\right)=\frac{p_{x}-E t^{n}}{\mid\left(p_{x}-E t^{n}, p_{y}\right)}$ :

$$
\widetilde{v}^{n}:=v\left(t^{n+1 / 2}\right)=v^{n}\left(x-\frac{\Delta t}{2} \frac{p_{x}-E t^{n}}{\left|\left(p_{x}-E t^{n}, p_{y}\right)\right|}\right) .
$$

(ii) Next, on $\left[t^{n}, t^{n+1}\right]$, we solve

$$
\partial_{t} v=e^{i \widetilde{S}\left(w, p_{x}\right) / \varepsilon}\left(\varepsilon \widetilde{\alpha}\left(w, x, p_{x}\right)+\widetilde{\beta}\left(w, x, p_{x}\right) v\right), \quad \partial_{t} w=1,
$$

with initial data $\left(\widetilde{v}^{n}, t^{n}\right)$. Obviously, the second equation gives $w(t)=t, t \in$ $\left[t^{n}, t^{n+1}\right]$. For the equation on $v$, we use an exponential scheme based on the following Duhamel formulation of $(5.10)$ (where for simplicity we omit the dependency in 
$x$ and $p_{x}$ of the unknowns and coefficients):

$$
\begin{aligned}
& v\left(t^{n+1}\right)=v\left(t^{n}\right)+\int_{t^{n}}^{t^{n+1}} e^{i \widetilde{S}(\tau) / \varepsilon}(\varepsilon \widetilde{\alpha}(\tau)+\widetilde{\beta}(\tau) v(\tau)) d \tau \\
& =v\left(t^{n}\right)+\int_{\widetilde{S}\left(t^{n}\right)}^{\widetilde{S}\left(t^{n+1}\right)} e^{i s / \varepsilon} \frac{1}{2\left|\left(p_{x}-E \widetilde{S}^{-1}(s), p_{y}\right)\right|}\left(\varepsilon \widetilde{\alpha}\left(\widetilde{S}^{-1}(s)\right)+\widetilde{\beta}\left(\widetilde{S}^{-1}(s)\right) v\left(\widetilde{S}^{-1}(s)\right)\right) d s \\
& =v\left(t^{n}\right)+\int_{\widetilde{S}\left(t^{n}\right)}^{\widetilde{S}\left(t^{n+1}\right)} e^{i s / \varepsilon} f\left(\widetilde{S}^{-1}(s), v\left(\widetilde{S}^{-1}(s)\right)\right) d s
\end{aligned}
$$

where we have denoted

$$
f(t, v)=\frac{1}{2\left|\left(p_{x}-E t, p_{y}\right)\right|}(\varepsilon \widetilde{\alpha}(t)+\widetilde{\beta}(t) v)
$$

and where we used the fact that $\partial_{t} \widetilde{S}\left(t, p_{x}\right)=\partial_{t} S\left(t, p_{x}-E t\right)=2\left|\left(p_{x}-E t, p_{y}\right)\right|$. Hence, our scheme for 5.10 is the following. In the first step, we make the prediction

$$
\widetilde{v}^{n+1 / 2}=\widetilde{v}^{n}+\int_{\widetilde{S}\left(t^{n}\right)}^{\widetilde{S}\left(t^{n+1 / 2}\right)} e^{i s / \varepsilon} f\left(t^{n}, \widetilde{v}^{n}\right) d s=\widetilde{v}^{n}+a_{n} f\left(t^{n}, \widetilde{v}^{n}\right),
$$

with

$$
a_{n}=\int_{\widetilde{S}\left(t^{n}\right)}^{\widetilde{S}\left(t^{n+1 / 2}\right)} e^{i s / \varepsilon} d s=-i \varepsilon\left(e^{i \widetilde{S}\left(t^{n+1 / 2}\right) / \varepsilon}-e^{i \widetilde{S}\left(t^{n}\right) / \varepsilon}\right) .
$$

Then in the second step, set

$$
\begin{aligned}
\widetilde{v}^{n+1}=\widetilde{v}^{n}+\int_{\widetilde{S}\left(t^{n}\right)}^{\widetilde{S}\left(t^{n+1}\right)} e^{i s / \varepsilon}( & f\left(t^{n+1 / 2}, \widetilde{v}^{n+1 / 2}\right) \\
& \left.\quad+\left(s-\widetilde{S}\left(t^{n+1 / 2}\right)\right) \frac{f\left(t^{n+1 / 2}, \widetilde{v}^{n+1 / 2}\right)-f\left(t^{n}, \widetilde{v}^{n}\right)}{\widetilde{S}\left(t^{n+1 / 2}\right)-\widetilde{S}\left(t^{n}\right)}\right) d s \\
= & \widetilde{v}^{n}+\left(b_{n}+c_{n}\right) f\left(t^{n+1 / 2}, \widetilde{v}^{n+1 / 2}\right)-c_{n} f\left(t^{n}, \widetilde{v}^{n}\right)
\end{aligned}
$$

with

$$
\begin{gathered}
b_{n}=\int_{\widetilde{S}\left(t^{n}\right)}^{\widetilde{S}\left(t^{n+1}\right)} e^{i s / \varepsilon} d s=-i \varepsilon\left(e^{i \widetilde{S}\left(t^{n+1}\right) / \varepsilon}-e^{i \widetilde{S}\left(t^{n}\right) / \varepsilon}\right), \\
c_{n}=\frac{1}{\widetilde{S}\left(t^{n+1 / 2}\right)-\widetilde{S}\left(t^{n}\right)} \int_{\widetilde{S}\left(t^{n}\right)}^{\widetilde{S}\left(t^{n+1}\right)} e^{i s / \varepsilon}\left(s-\widetilde{S}\left(t^{n+1 / 2}\right)\right) d s \\
=\varepsilon^{2} \frac{e^{i \widetilde{S}\left(t^{n+1}\right) / \varepsilon}-e^{i \widetilde{S}\left(t^{n}\right) / \varepsilon}}{\widetilde{S}\left(t^{n+1 / 2}\right)-\widetilde{S}\left(t^{n}\right)}-i \frac{\varepsilon}{\widetilde{S}\left(t^{n+1 / 2}\right)-\widetilde{S}\left(t^{n}\right)} \times \\
\left(e^{i \widetilde{S}\left(t^{n+1}\right) / \varepsilon}\left(\widetilde{S}\left(t^{n+1}\right)-\widetilde{S}\left(t^{n+1 / 2}\right)\right)+e^{i \widetilde{S}\left(t^{n}\right) / \varepsilon}\left(\widetilde{S}\left(t^{n+1 / 2}\right)-\widetilde{S}\left(t^{n}\right)\right)\right) .
\end{gathered}
$$

(iii) Finally, on $\left[t^{n+1 / 2}, t^{n+1}\right]$, (5.8) with initial data $\left(\widetilde{v}^{n+1}, w\left(t^{n+1}\right)\right)$ can be solved exactly with the characteristics:

$$
v^{n+1}=\widetilde{v}^{n+1}\left(x-\frac{\Delta t}{2} \frac{p_{x}-E t^{n+1}}{\left|\left(p_{x}-E t^{n+1}, p_{y}\right)\right|}\right) .
$$

The global error of this semi-discrete approximation of (5.1) has two contributions. First, the error of the Strang splitting scheme is $\mathcal{O}\left(\Delta t^{2}\right)$ uniform in $\varepsilon$ (as we 
shall see in the Proposition 5.2), since $S$ is independent of $x$ and since, by (5.2), (5.3) the second derivatives in $x$ of $\alpha, \beta$ and $u$ are uniformly bounded. Secondly, the error of the exponential scheme is $\mathcal{O}\left(\Delta t^{2}\right)$, uniform in $\varepsilon$ (as we shall see in the Proposition 5.1. . One of the key arguments lies in the uniform boundedness of the second derivatives in $t$ and $p_{x}$ of $\alpha, \beta$ and $u$.

Let us start with the error on the exponential scheme (5.13).

Proposition 5.1. Let $v\left(t^{n}\right)$ be the solution at $t=t^{n}$ of (5.10) and $\widetilde{v}^{n}$ is the time approximation of $v\left(t^{n}\right)$ given by the second order exponential scheme (5.12)-(5.13). Under assumptions (5.2)-(5.3), the exponential scheme to (5.10) is second order accurate uniformly in $\varepsilon$ : there exists $C>0$ independent of $\varepsilon$ such that

$$
\left\|\widetilde{v}^{n}-v\left(t^{n}\right)\right\|_{L_{x, p_{x}}^{\infty}} \leq C \Delta t^{2}, \forall n \Delta t \leq T_{0} .
$$

Proof. We consider (5.11). Using a Taylor expansion (with integral remainder) of $V\left(\widetilde{S}^{-1}(s)\right):=f\left(\widetilde{S}^{-1}(s), v\left(\widetilde{S}^{-1}(s)\right)\right)$ at $s=\left(\widetilde{S}\left(t^{n+1 / 2}\right)\right)$, we get

$$
\begin{aligned}
V\left(\widetilde{S}^{-1}(s)\right)= & V\left(t^{n+1 / 2}\right)+\frac{\left(s-\widetilde{S}\left(t^{n+1 / 2}\right)\right)}{\widetilde{S}\left(t^{n+1 / 2}\right)-\widetilde{S}\left(t^{n}\right)}\left[V\left(t^{n+1 / 2}\right)-V\left(t^{n}\right)\right] \\
& +\left(s-\widetilde{S}\left(t^{n+1 / 2}\right)\right)\left(\widetilde{S}\left(t^{n+1 / 2}\right)-\widetilde{S}\left(t^{n}\right)\right) \times \\
& \int_{0}^{1}(1-t)\left(V \circ \widetilde{S}^{-1}\right)^{\prime \prime}\left(\widetilde{S}\left(t^{n+1 / 2}\right)+t\left(\widetilde{S}\left(t^{n}\right)-\widetilde{S}\left(t^{n+1 / 2}\right)\right)\right) d t \\
+ & \left(s-\widetilde{S}\left(t^{n+1 / 2}\right)\right)^{2} \int_{0}^{1}(1-t)\left(V \circ \widetilde{S}^{-1}\right)^{\prime \prime}\left(\widetilde{S}\left(t^{n+1 / 2}\right)+t\left(s-\widetilde{S}\left(t^{n+1 / 2}\right)\right)\right) d t .
\end{aligned}
$$

First, thanks to Theorem 4.1. the second time derivatives of the solution are uniformly bounded ; second, the function $S$ is also smooth with respect to $\varepsilon$, so are $\alpha$, $\beta$. Hence, the solution $v(t)$ satisfies the scheme (5.13) up to the following remainder term $\mathcal{R}$

$$
\begin{aligned}
\mathcal{R}= & \left(s-\widetilde{S}\left(t^{n+1 / 2}\right)\right)\left(\widetilde{S}\left(t^{n+1 / 2}\right)-\widetilde{S}\left(t^{n}\right)\right) \times \\
& \int_{0}^{1}(1-t)\left(V \circ \widetilde{S}^{-1}\right)^{\prime \prime}\left(\widetilde{S}\left(t^{n+1 / 2}\right)+t\left(\widetilde{S}\left(t^{n}\right)-\widetilde{S}\left(t^{n+1 / 2}\right)\right)\right) d t \\
& +\left(s-\widetilde{S}\left(t^{n+1 / 2}\right)\right)^{2} \int_{0}^{1}(1-t)\left(V \circ \widetilde{S}^{-1}\right)^{\prime \prime}\left(\widetilde{S}\left(t^{n+1 / 2}\right)+t\left(s-\widetilde{S}\left(t^{n+1 / 2}\right)\right)\right) d t .
\end{aligned}
$$

Then, the remainder satisfies the following estimates

$$
\begin{aligned}
|\mathcal{R}| & \leq C \int_{\widetilde{S}\left(t^{n}\right)}^{\widetilde{S}\left(t^{n+1}\right)}\left[\left|s-\widetilde{S}\left(t^{n+1 / 2}\right)\right|\left(\widetilde{S}\left(t^{n+1 / 2}\right)-\widetilde{S}\left(t^{n}\right)\right)+\left|s-\widetilde{S}\left(t^{n+1 / 2}\right)\right|^{2}\right] d s \\
& \leq C \Delta t^{3},
\end{aligned}
$$

since the derivatives of $\alpha, \beta$ and $u$ are uniformly bounded. Define the error $\mathcal{E}^{n+1}:=$ $\left\|v\left(t^{n+1}\right)-\widetilde{v}^{n+1}\right\|_{L_{x, p_{x}}^{\infty}}$. Then, the error $\mathcal{E}^{n+1}$ satisfies the following estimate

$$
\mathcal{E}^{n+1} \leq \mathcal{E}^{n}(1+C \Delta t)+C \Delta t \mathcal{E}^{n+1 / 2}+C \Delta t^{3} .
$$

To get an estimate on the error $\mathcal{E}^{n+1 / 2}$ associated with the prediction step (5.12), we perform a Taylor expansion of $V\left(\widetilde{S}^{-1}(s)\right):=f\left(\widetilde{S}^{-1}(s), v\left(\widetilde{S}^{-1}(s)\right)\right)$ at $s=\widetilde{S}\left(t^{n}\right)$

$$
V\left(\widetilde{S}^{-1}(s)\right)=V\left(t^{n}\right)+\int_{\widetilde{S}\left(t^{n}\right)}^{\widetilde{S}\left(t^{n+1 / 2}\right)}\left(V \circ \widetilde{S}^{-1}\right)^{\prime}(s) d s .
$$


Then, the error $\mathcal{E}^{n+1 / 2}$ performed after the prediction step $(5.12)$ satisfies $\mathcal{E}^{n+1 / 2} \leq$ $\mathcal{E}^{n}(1+C \Delta t)+C \Delta t^{2}$ so that (5.15) becomes

$$
\mathcal{E}^{n+1} \leq \mathcal{E}^{n}(1+C \Delta t)+C \Delta t^{3},
$$

which produces uniform second order accuracy at final time $T=N \Delta t$ using the discrete Gronwall Lemma.

Then, we prove that the Strang splitting is second order, uniformly in $\varepsilon$.

Proposition 5.2. Let $v\left(t^{n}\right)$ be the solution at $t=t^{n}$ of (5.4) and $v^{n}$ be the time approximation of $v\left(t^{n}\right)$ given by the Strang splitting (5.9)-(5.13)-(5.14). Under the assumptions (5.2)-(5.3), the Strang splitting applied to (5.5) is second order accurate uniformly in $\varepsilon$ : there exists $C>0$ independent of $\varepsilon$ such that

$$
\left\|v^{n}-v\left(t^{n}\right)\right\|_{L_{x, p_{x}}^{\infty}} \leq C \Delta t^{2}, \quad \forall n \Delta t \leq T_{0} .
$$

Proof. Following [14, to estimate the local error of the Strang splitting, we need to compute the following brackets $[f,[f, g]](y)$ and $[[f, g], g](y)$ where $f(y), g(y) \in \mathbb{R}^{2}$ are two vector fields and $[f, g](y):=\left(\partial_{y} f\right)(y) g(y)-\left(\partial_{y} g\right)(y) f(y)$ where we recall $y=(v, w)$. For the splitting (5.6)-(5.7) considered above,

$f(y):=\left(\begin{array}{l}-a(w, p) \partial_{x} v \\ 0\end{array}\right)$ and $g(y)=\left(\begin{array}{l}e^{i \widetilde{S}\left(w, p_{x}\right) / \varepsilon}\left(\varepsilon \widetilde{\alpha}\left(w, x, p_{x}\right)+\widetilde{\beta}\left(w, x, p_{x}\right) v\right) \\ 1\end{array}\right)$.

Hence, we compute the following quantities

$$
\partial_{y} f(y)=\left(\begin{array}{ll}
-a \partial_{x}(\cdot) & -\partial_{w} a \partial_{x} v \\
0 & 0
\end{array}\right) \quad \text { and } \quad \partial_{y} g(y)=\left(\begin{array}{ll}
e^{i \widetilde{S} / \varepsilon} \widetilde{\beta} & \partial_{w} g_{1}(y) \\
0 & 0
\end{array}\right),
$$

where $\partial_{w} g_{1}(y)$ denotes the $w$-derivative of the first component of $g$

$$
\partial_{w} g_{1}(y)=e^{i \widetilde{S} / \varepsilon} i \partial_{w} S\left(\widetilde{\alpha}+\frac{v}{\varepsilon} \widetilde{\beta}\right)+e^{i \widetilde{S} / \varepsilon}\left(\varepsilon \partial_{w} \widetilde{\alpha}+v \partial_{w} \widetilde{\beta}\right) .
$$

We have

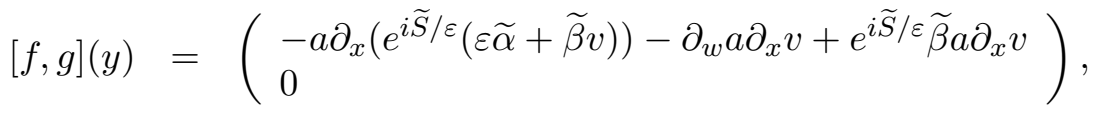

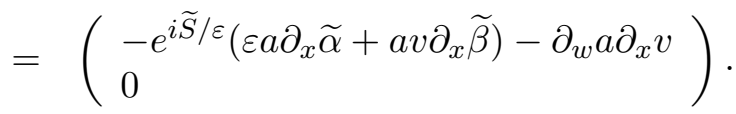

Thanks to the estimates (5.2) and (5.3), this term is uniformly bounded with respect to $\varepsilon$. Now, we consider $[f,[f, g]]=\left(\partial_{y} f\right)(y)[f, g](y)-\left(\partial_{y}[f, g]\right)(y) f(y)$. First, we compute $\partial_{y}[f, g](y) f(y)$

$$
\begin{aligned}
\partial_{y}[f, g](y) f(y) & =\left(\begin{array}{ll}
-e^{i \widetilde{S} / \varepsilon} a \partial_{x} \widetilde{\beta}-\partial_{w} a \partial_{x}(\cdot) & \partial_{w}[f, g]_{1}(y) \\
0 & 0
\end{array}\right)\left(\begin{array}{l}
-a \partial_{x} v \\
0
\end{array}\right) \\
& =\left(\begin{array}{l}
e^{i \widetilde{S} / \varepsilon} \partial_{x} \widetilde{\beta} a^{2} \partial_{x} v+a \partial_{w} a \partial_{x}^{2} v \\
0
\end{array}\right),
\end{aligned}
$$

where $\partial_{w}[f, g]_{1}(y)$ denotes the $w$-derivative of the first component of $[f, g]$

$$
\begin{aligned}
\partial_{w}[f, g]_{1}(y)= & -e^{i \widetilde{S} / \varepsilon} i \partial_{w} S\left(a \partial_{x} \widetilde{\alpha}+\frac{v}{\varepsilon} a \partial_{x} \widetilde{\beta}\right) \\
& -e^{i \widetilde{S} / \varepsilon} \partial_{w}\left(\varepsilon a \partial_{x} \widetilde{\alpha}+a v \partial_{x} \widetilde{\beta}\right)-\partial_{w}^{2} a \partial_{x} v .
\end{aligned}
$$


Second we compute $\left(\partial_{y} f\right)(y)[f, g](y)$

$$
\begin{aligned}
\left(\partial_{y} f\right)(y)[f, g](y) & =\left(\begin{array}{ll}
-a \partial_{x}(\cdot) & -\partial_{w} a \partial_{x} v \\
0 & 0
\end{array}\right)\left(\begin{array}{l}
-e^{i \widetilde{S} / \varepsilon}\left(\varepsilon a \partial_{x} \widetilde{\alpha}+a v \partial_{x} \widetilde{\beta}\right)-\partial_{w} a \partial_{x} v \\
0
\end{array}\right) \\
& =\left(\begin{array}{ll}
a e^{i \widetilde{S} / \varepsilon} \partial_{x}\left(\varepsilon a \partial_{x} \widetilde{\alpha}+a v \partial_{x} \widetilde{\beta}\right)+a \partial_{w} a \partial_{x}^{2} v \\
0
\end{array}\right) .
\end{aligned}
$$

Finally, we compute $[[f, g], g]$

$$
\begin{aligned}
{[[f, g], g] } & =\partial_{y}[f, g](y) g(y)-\partial_{y} g(y)[f, g](y) \\
& =\partial_{v}[f, g]_{1} g_{1}+\partial_{w}[f, g]_{1} g_{2}-\partial_{v} g_{1}[f, g]_{1}-\partial_{w} g_{1}[f, g]_{2}
\end{aligned}
$$

In the above expressions, the terms $\partial_{v}[f, g]_{1} g_{1}$ and $\partial_{v} g_{1}[f, g]_{1}$ are clearly uniformly bounded. $\partial_{w}[f, g]_{1} g_{2}$ and $\partial_{w} g_{1}[f, g]_{2}$, however, have to be discussed since they involve $v / \varepsilon$ terms (see (5.18) and (5.16). But we proved in Proposition 3.1 that $v / \varepsilon$ is uniformly bounded, then we conclude that the term $[[f, g], g]$ is uniformly bounded. Thanks to the previous estimates, the quantities (5.17), (5.19) and (5.20) are uniformly bounded, which ensures that the Strang splitting is uniformly second order accurate in time.

\subsection{Numerical scheme for the full system (3.4).}

To summarize, model (3.4) can be solved from its micro-macro formulation as follows

- solve the macro system (3.5) with initial conditions (3.9)- $(3.10)$.

This enables to get $\left(F_{0}^{ \pm}(t, x, p), G_{0}(t, x, p)\right)$.

- solve the micro equations (3.7) with initial conditions (3.11)-(3.12).

This enables to get $\left(h^{ \pm}(t, x, p), h(t, x, p)\right)$.

- solve the phase equation (3.8) using (3.36).

- reconstruct the solution $\left(f^{ \pm}, g\right)(t, x, p)$ to the original problem (3.4) using the micro-macro decomposition (3.14)-3.15.

This micro-macro formulation is the basis on which we designed a uniformly accurate (UA) numerical scheme. Note in particular tha, unlike in [6], here t no additional variable $\tau$ is used. Some numerical experiments are reported in the following section to demonstrate the efficiency of our approach.

\section{Numerical results}

We now present some numerical experiments for model (3.4). For the first test, the initial data are:

$$
\begin{gathered}
f_{+}\left(0, x, p_{x}\right)=\frac{4}{\pi} e^{-4(x+2)^{2}-4\left(p_{x}-1.3\right)^{2}}, \\
f_{-}\left(0, x, p_{x}\right)=0, \\
f_{i}\left(0, x, p_{x}\right)=\frac{2(1+i)}{\pi} e^{-4(x+2)^{2}-4\left(p_{x}-1.3\right)^{2}},
\end{gathered}
$$

and $p_{y}=0.5$ is a parameter in the model, $E=4$, while the domain in $\left(x, p_{x}\right)$ is $[-2 \pi, 2 \pi]^{2}$. The number of grid points in $x$ is $N_{x}=128$ and in $p_{x}$ is $N_{p}=128$. The final time is $t_{f}=0.2$. In Figure 1, we have plotted in logarithmic scales the $L^{1}$ error on $f_{+}, f_{-}$and $f_{i}$ as a function of $\Delta t$, for 7 values of $\varepsilon$. In Figure 2 , we have plotted the error as a function of $\varepsilon$ for 6 values of $\Delta t$. It appears on these figures 
that our scheme is Uniformly Accurate of order 2. Note that the reference solution is computed using a direct splitting method for (3.1) with $\Delta t=10^{-4}, N_{x}=512$ and $N_{p}=2048$ when $\varepsilon>10^{-2}$ whereas for $\varepsilon \leq 10^{-2}$ the reference solution is obtained by solving the micro-macro model derived in Proposition 3.1 with $\Delta t=10^{-4}, N_{x}=512$ and $N_{p}=512$. Note that the micro-macro numerical solutions $u$ are computed at the final time by using the staggered mesh strategy in $p_{x}$ (see Section 5 for more details).

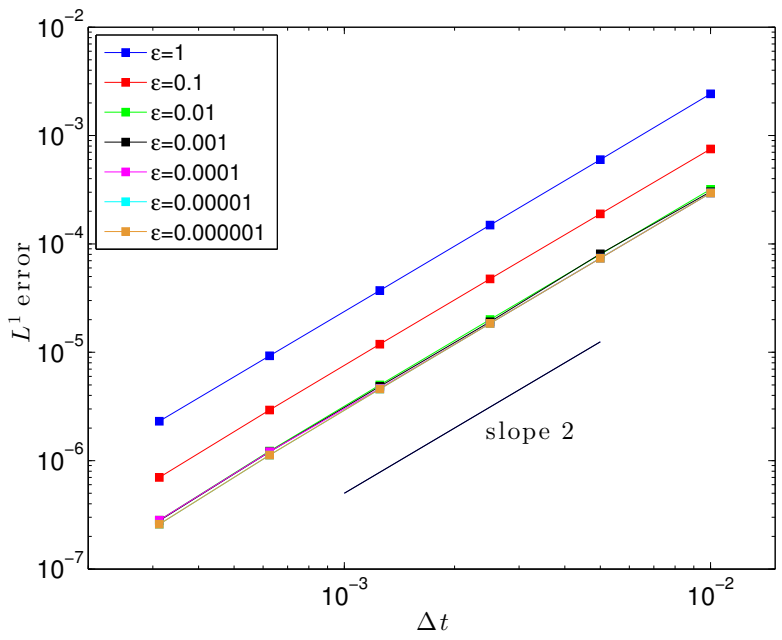

Figure 1: Error as a function of $\Delta t$ for $\varepsilon=10^{-k}, k=0, \ldots, 6$.

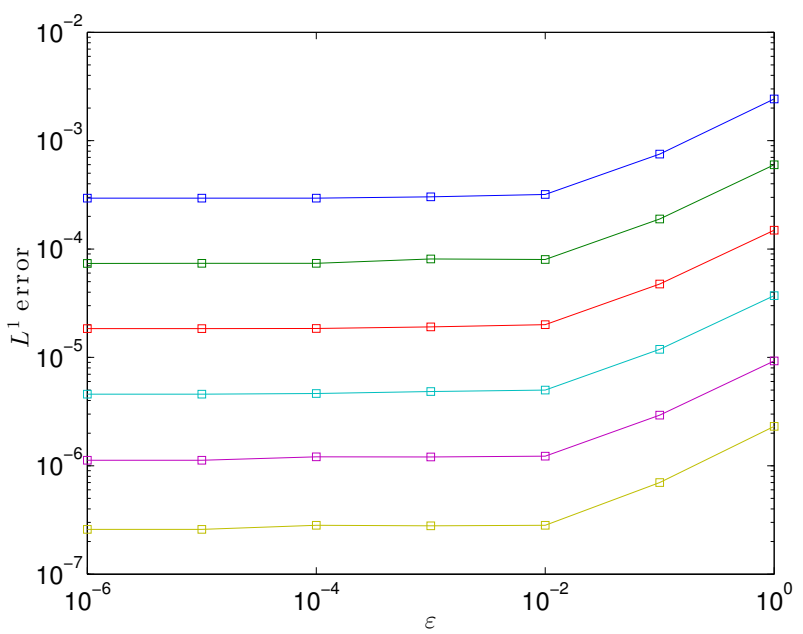

Figure 2: Error as a function of $\varepsilon$ for $\Delta t=2^{-k} \times 10^{-2}, k=0, \ldots, 5$. Lower curves correspond to bigger $k$.

For the next simulation, the number of grid points in $x$ is $N_{x}=64$ and in $p_{x}$ is $N_{p}=128$. The time step is $\Delta t=0.02$, the final time is $t_{f}=1$ and $\varepsilon=0.005$. 
The initial data, $p_{y}$ and $E$ are the same as above. In Figure 3 , we have represented $\Re f_{i}\left(t_{f},-2.16, p_{x}\right)$ as a function of $p_{x}$ : in blue, the reference solutions and in red the solutions computed with the above parameters. In Figure 4. we have represented $\Re f_{i}(t,-2.16,-1.18)$ as a function of time: in blue, the reference solutions and in red the solutions computed with the above parameters. The solution in red fits well with the one in blue, even if the mesh in $p_{x}$ or in $t$ is coarse and does not resolve the oscillations. Here, the micro-macro numerical solutions $u$ are computed using an interpolation in $p_{x}$ (see Section 5 for more details).
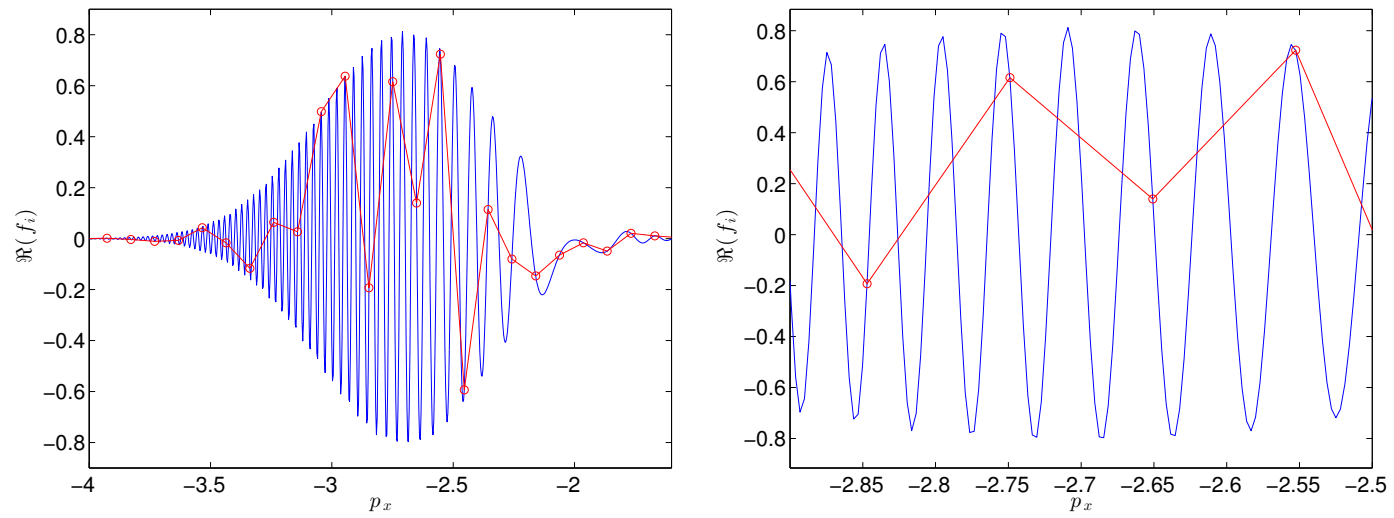

Figure 3: For $\varepsilon=0.005: \Re\left(f_{i}\right)\left(t_{f},-2.16, p_{x}\right)$ as a function of $p_{x}$. The right figure is a zoom of the left figure.
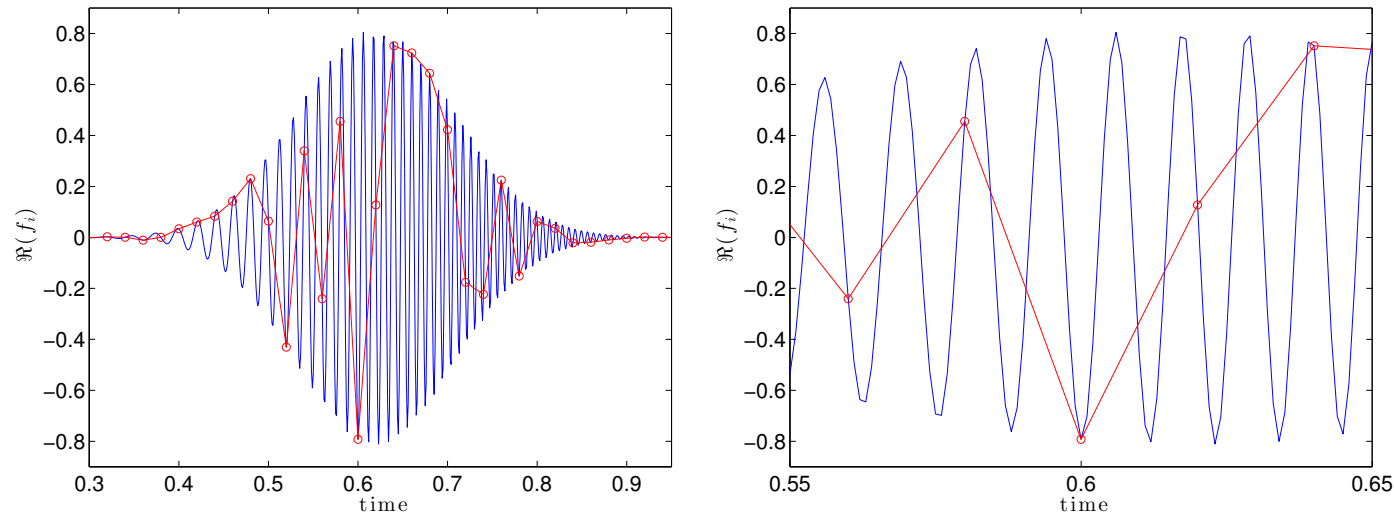

Figure 4: For $\varepsilon=0.005: \Re\left(f_{i}\right)(t,-2.16,-1.18)$ as a function of $t$. The right figure is a zoom of the left figure.

We present the last test, for which the initial data are

$$
f_{+}\left(0, x, p_{x}\right)=\frac{4}{\pi} e^{-4(x+2)^{2}-4\left(p_{x}-1.3\right)^{2}}, \quad f_{-}\left(0, x, p_{x}\right)=0, \quad f_{i}\left(0, x, p_{x}\right)=0 .
$$


and $p_{y}=0.25, E=4$, the domain in $\left(x, p_{x}\right)$ being the same as above. The number of grid points in $x$ is $N_{x}=64$ and in $p_{x}$ is $N_{p}=128$. The time step is $\Delta t=0.005$ and the final time is $t_{f}=1$. We observe the transition of mass between $f_{+}$and $f_{-}$:

$$
T=\frac{\int f_{-}\left(t_{f}, x, p_{x}\right) d x d p_{x}}{\int f_{+}\left(0, x, p_{x}\right) d x d p_{x}}
$$

and have plotted in Figure 5 in logarithmic scales the transition rate as a function of $\varepsilon$, compared with the theoretical Landau-Zener transition rate

$$
T_{\text {Landau-Zener }}=\exp \left(-\frac{\pi p_{y}^{2}}{\varepsilon E}\right) .
$$

The two curves coincide well which shows two interesting facts: this semiclassical model for transport in graphene reproduces very well the Landau-Zener transition rate and our scheme is able to capture this coefficient for a wide range of $\varepsilon$, with fixed numerical parameters.

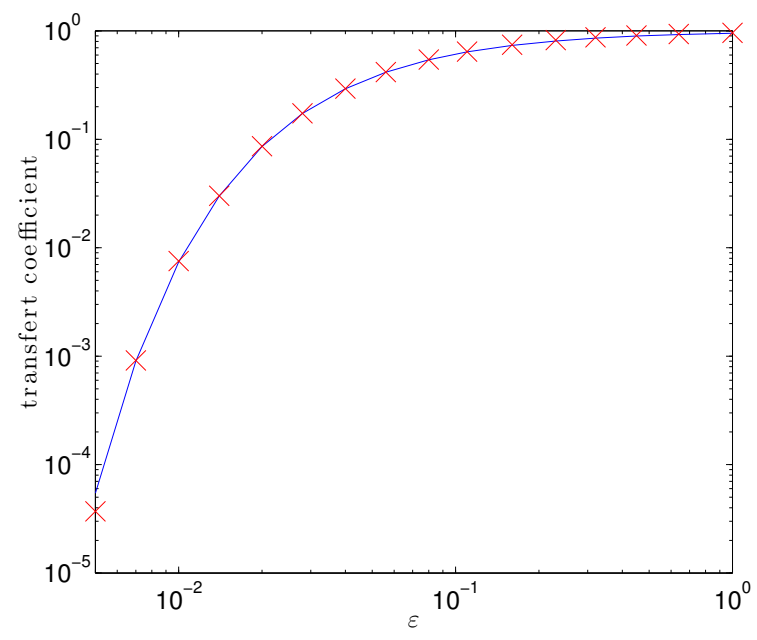

Figure 5: The Landau-Zener probability. Red crosses=numerically computed transfert coefficient. Blue curve=theoretical coefficient.

\section{Conclusion}

In this paper, we propose a micro-macro decomposition based numerical approach for the one space dimensional semiclassical kinetic graphene model introduced in [20]. Comparing with the nonlinear geometric optics method based numerical method for highly oscillatory transport equation developed by the authors in [6], this new method does not increase the dimension of computational domain, yet can still capture numerically the oscillatory space-time quantum solution pointwisely even without numerically resolving the frequency. The underlying micromacro equations have been proved to be smooth (up to certain order of derivatives) solutions with respect to the frequency, and the proposed numerical time discretization are proved to have a uniform accuracy for a scalar model equation exhibiting 
the same oscillatory behavior. We carried out several numerical experiments to verify the theory.

In the future, we will extend the method for the two-space dimensional model.

\section{Appendix A. Non-homogeneous $E(x)$ case}

In the case $E=E(x)$, we need to compute additional terms in the micro model derived above in the homogeneous case. Let us recall the definition of $\mathcal{E}^{ \pm}$

$$
\mathcal{E}^{ \pm}=2|p| \pm \frac{p_{x}}{|p|} \partial_{x} S
$$

Note that the phase now depends on $x$.

In this case, the Chapman-Enskog expansion now reads

$$
\begin{aligned}
& G=G_{0}+G_{1}=G_{0}-\frac{\varepsilon p_{y} \bar{p} E}{4|p|^{4}} e^{i \tau}\left(F_{0}^{+}-F_{0}^{-}\right), \\
& F^{+}=F_{0}^{+}+F_{1}^{+}=F_{0}^{+}+\frac{\varepsilon p_{y} E}{\mathcal{E}^{+}|p|^{3}} \Im\left(i p e^{-i \tau} G_{0}\right), \\
& F^{-}=F_{0}^{-}+F_{1}^{-}=F_{0}^{-}-\frac{\varepsilon p_{y} E}{\mathcal{E}^{-}|p|^{3}} \Im\left(i p e^{-i \tau} G_{0}\right),
\end{aligned}
$$

where $F_{0}^{ \pm}=\Pi F^{ \pm}, G_{0}=\Pi G$. The same computations as in the homogeneous case leads to the following macro model for $\left(F_{0}^{ \pm}, G_{0}\right)$

$$
\begin{aligned}
\partial_{t} F_{0}^{+}+\mathcal{T}^{+} F_{0}^{+} & =0 \\
\partial_{t} F_{0}^{-}+\mathcal{T}^{-} F_{0}^{-} & =0 \\
\partial_{t} G_{0}-E \partial_{p_{x}} G_{0} & =-i \frac{p_{y}}{|p|^{2}} E G_{0}-i \varepsilon \frac{\bar{p} p_{y}^{2} E^{2}}{2|p|^{6}}\left(\frac{1}{\mathcal{E}^{+}}+\frac{1}{\mathcal{E}^{-}}\right) \Pi\left(e^{i \tau} \Im\left(i p e^{-i \tau} G_{0}\right)\right),
\end{aligned}
$$

with the following initial conditions

$$
\begin{aligned}
F^{ \pm}(0, x, p) & =f_{\text {in }}^{ \pm}(x, p) \mp \frac{\varepsilon p_{y} E}{\mathcal{E}^{ \pm}|p|^{3}} \Im\left(i p f_{\text {in }}(x, p)\right), \\
G_{0}(0, x, p) & =f_{\text {in }}(x, p)+\frac{\varepsilon p_{y} \bar{p} E}{4|p|^{4}}\left(f_{\text {in }}^{+}(x, p)-f_{\text {in }}^{-}(x, p)\right), \\
h^{ \pm}(0, x, p) & \text { and } \quad h(0, x, p) \text { such that } 3.15 \text { holds at } t=0, \\
S(0, x, p) & =0 .
\end{aligned}
$$

Micro equation on $h^{+}$. From the Chapman-Enskog expansion (A.1) and relations (3.16), we can write the following micro-macro decomposition of the original unknown

$$
\begin{aligned}
f^{ \pm}(t, x, p) & =F_{0}^{ \pm}(t, x, p) \pm \frac{\varepsilon p_{y} E}{|p|^{3} \mathcal{E}^{ \pm}} \Im\left(i p e^{-i S / \varepsilon} G_{0}(t, x, p)\right)+h^{ \pm}(t, x, p), \\
g(t, x, p) & =G_{0}(t, x, p)-\frac{\varepsilon p_{y} \bar{p} E}{4|p|^{4}}\left(F_{0}^{+}(t, x, p)-F_{0}^{-}(t, x, p)\right) e^{i S / \varepsilon}+h(t, x, p) .
\end{aligned}
$$


These relations impose in particular that $h^{ \pm}$and $h$ are of order $\varepsilon^{2}$. To derive an equation for $h^{ \pm}$, we apply the operator $\left(\partial_{t}+\mathcal{T}^{ \pm}\right)$to (A.7). For $h^{+}$, we get

$$
\left(\partial_{t}+\mathcal{T}^{+}\right) h^{+}=\left(\partial_{t}+\mathcal{T}^{+}\right) f^{+}-\left(\partial_{t}+\mathcal{T}^{+}\right) F_{0}^{+}-\left(\partial_{t}+\mathcal{T}^{+}\right)\left(\frac{\varepsilon p_{y} E}{|p|^{3} \mathcal{E}^{+}} \Im\left(i p e^{-i S / \varepsilon} G_{0}\right)\right),
$$

and we need to compute the last term (which we called $\mathcal{C}$ given by $(3.28)$ in the homogeneous case) which in the present non homogeneous case reads

$$
\begin{aligned}
\mathcal{C}= & \mathcal{B}^{+} \Im\left(i p e^{-i S / \varepsilon} G_{0}\right)+\left(\frac{\varepsilon p_{y} E}{|p|^{3} \mathcal{E}^{+}}\right) \Im\left(\left(\partial_{t}+\mathcal{T}^{+}\right)\left(i p G_{0}\right) e^{-i S / \varepsilon}\right) \\
& +\left(\frac{\varepsilon p_{y} E}{|p|^{3} \mathcal{E}^{+}}\right) \Im\left(i p G_{0} \frac{-i}{\varepsilon} e^{-i S / \varepsilon}\left(\partial_{t}+\mathcal{T}^{+}\right) S\right)
\end{aligned}
$$

where $\mathcal{B}^{+}$is now given by

$$
\begin{aligned}
\mathcal{B}^{+}:= & \left(\partial_{t}+\mathcal{T}^{+}\right)\left(\frac{\varepsilon p_{y} E}{|p|^{3} \mathcal{E}^{+}}\right)=\partial_{t}\left(\frac{\varepsilon p_{y} E}{|p|^{3} \mathcal{E}^{+}}\right)+\frac{p_{x}}{|p|} \partial_{x}\left(\frac{\varepsilon p_{y} E}{|p|^{3} \mathcal{E}^{+}}\right)-E \partial_{p_{x}}\left(\frac{\varepsilon p_{y} E}{|p|^{3} \mathcal{E}^{+}}\right) \\
= & -\frac{\varepsilon p_{x} p_{y} E \partial_{x} E}{|p|^{4} \mathcal{E}^{+, 2}} \partial_{p_{x}} S-\frac{\varepsilon p_{x} p_{y} E^{2}}{|p|^{4} \mathcal{E}^{+, 2}} \partial_{x, p_{x}}^{2} S \\
& +\frac{\varepsilon p_{x} p_{y}}{|p|^{4} \mathcal{E}^{+, 2}}\left(\mathcal{E}^{+} \partial_{x} E-E\left(p_{x} /|p| \partial_{x}^{2} S\right)\right) \\
& \left.-\frac{\varepsilon p_{y}}{|p|^{6} \mathcal{E}^{+, 2}}\left(E \partial_{p_{x}} E|p|^{3} \mathcal{E}^{+}-8 p_{x}|p|^{2} E^{2}-\left(|p|^{2}+2 p_{x}^{2}\right) E^{2} \partial_{x} S-E^{2} p_{x}|p|^{2} \partial_{x, p_{x}}^{2} S\right]\right) \\
= & \frac{\varepsilon p_{y}}{|p|^{3} \mathcal{E}^{+}}\left(\frac{p_{x}}{|p|} \partial_{x} E-E \partial_{p_{x}} E\right)-\frac{\varepsilon p_{x} p_{y} E \partial_{x} E \partial_{p_{x}} S}{|p|^{4} \mathcal{E}^{+, 2}} \\
& -\frac{\varepsilon p_{x}^{2} p_{y} E \partial_{x}^{2} S}{|p|^{5} \mathcal{E}^{+, 2}}+\frac{\varepsilon p_{y} E^{2}}{|p|^{4} \mathcal{E}^{+, 2}}\left(8 p_{x}+\partial_{x} S\left(1-2 p_{x}^{2} /|p|^{2}\right)\right) .
\end{aligned}
$$

Moreover, we can compute $\mathcal{D}^{+}:=\Im\left(\left(\partial_{t}+\mathcal{T}^{+}\right)\left(i p G_{0}\right) e^{-i S / \varepsilon}\right)$ (given by 3.30 in the homogeneous case)

$$
\begin{aligned}
\mathcal{D}^{+}= & -E \Im\left(i G_{0} e^{-i S / \varepsilon}\right)+\frac{p_{y} E}{|p|^{2}} \Im\left(p G_{0} e^{-i S / \varepsilon}\right) \\
& +\frac{p_{x}}{|p|} \Im\left(i p \partial_{x} G_{0} e^{-i S / \varepsilon}\right)+\frac{\varepsilon p_{y}^{2} E^{2}}{|p|^{3} \mathcal{E}^{+} \mathcal{E}^{-}} \Im\left(p G_{0} e^{-i S / \varepsilon}\right),
\end{aligned}
$$

so that, the micro equation on $h^{+}$in the case $E(x)$ reads

$$
\begin{aligned}
\left(\partial_{t}+\mathcal{T}^{+}\right) h^{+}= & R[h]+\mathcal{B}^{+} R\left[i G_{0}\right]+\frac{\varepsilon E}{2|p|} R\left[i G_{0} / p\right] \\
& -\frac{\varepsilon p_{y} E}{2|p|^{3}} R\left[G_{0}\right]\left(1+\frac{2 \varepsilon p_{y} E}{\mathcal{E}^{+, 2} \mathcal{E}^{-}}\right)-\frac{\varepsilon p_{x}}{2|p|^{2}} R\left[i \partial_{x} G_{0}\right]
\end{aligned}
$$

with $R[\cdot]=\frac{p_{y}}{|p|^{3}} E \Im\left(p e^{-i S / \varepsilon}.\right)$ and $\mathcal{B}^{+}$is given by A.11. 
Micro equation on $h^{-}$. Defining as before the quantity $\mathcal{B}^{-}$as

$$
\begin{aligned}
\mathcal{B}^{-}:= & \left(\partial_{t}+\mathcal{T}^{-}\right)\left(\frac{\varepsilon p_{y} E}{|p|^{3} \mathcal{E}^{-}}\right)=\partial_{t}\left(\frac{\varepsilon p_{y} E}{|p|^{3} \mathcal{E}^{-}}\right)-\frac{p_{x}}{|p|} \partial_{x}\left(\frac{\varepsilon p_{y} E}{|p|^{3} \mathcal{E}^{-}}\right)-E \partial_{p_{x}}\left(\frac{\varepsilon p_{y} E}{|p|^{3} \mathcal{E}^{-}}\right) \\
= & -\frac{\varepsilon p_{x} p_{y} E \partial_{x} E}{|p|^{4} \mathcal{E}^{-, 2}} \partial_{p_{x}} S-\frac{\varepsilon p_{x} p_{y} E^{2}}{|p|^{4} \mathcal{E}^{-, 2}} \partial_{x, p_{x}}^{2} S \\
& -\frac{\varepsilon p_{x} p_{y}}{|p|^{4} \mathcal{E}^{-, 2}}\left(\mathcal{E}^{-} \partial_{x} E+E\left(p_{x} /|p| \partial_{x}^{2} S\right)\right) \\
& \left.-\frac{\varepsilon E p_{y}}{|p|^{6} \mathcal{E}^{-, 2}}\left(\partial_{p_{x}} E|p|^{3} \mathcal{E}^{-}-8 p_{x}|p|^{2} E+\left(|p|^{2}+2 p_{x}^{2}\right) E \partial_{x} S+E p_{x}|p|^{2} \partial_{x, p_{x}}^{2} S\right]\right) .
\end{aligned}
$$

The micro equation on $h^{-}$in the case $E(x)$ then reads

$$
\begin{aligned}
\left(\partial_{t}+\mathcal{T}^{-}\right) h^{-}= & -R[h]+\mathcal{B}^{-} R\left[i G_{0}\right]-\frac{\varepsilon E}{2|p|} R\left[i G_{0} / p\right] \\
& +\frac{\varepsilon p_{y} E}{2|p|^{3}} R\left[G_{0}\right]\left(1+\frac{2 \varepsilon p_{y} E}{\mathcal{E}^{-, 2} \mathcal{E}^{+}}\right)-\frac{\varepsilon p_{x}}{2|p|^{2}} R\left[i \partial_{x} G_{0}\right]
\end{aligned}
$$

with $R[\cdot]=\frac{p_{y}}{|p|^{3}} E \Im\left(p e^{-i S / \varepsilon}.\right)$ and $\mathcal{B}^{-}$is given by A.13.

Micro equation on $h$. The micro equation on $h$ can be easily derived in the non homogeneous case

$$
\begin{aligned}
\left(\partial_{t}-E \partial_{p_{x}}\right) h= & \frac{3 \varepsilon p_{x} p_{y} \bar{p} E^{2}}{4|p|^{6}} e^{i S / \varepsilon}\left(F_{0}^{+}-F_{0}^{-}\right)-\frac{i p_{y} E}{|p|^{2}} h \\
& -\frac{i p_{y} \bar{p} E}{2|p|^{3}} e^{i S / \varepsilon}\left(h^{+}-h^{-}\right)-\varepsilon \frac{i p_{y}^{2} \bar{p}^{2} E^{2}}{|p|^{5} \mathcal{E}^{+} \mathcal{E}^{-}} e^{2 i S / \varepsilon} \bar{G}_{0} \\
& -\frac{\varepsilon p_{y} p_{x} \bar{p} E}{4|p|^{5}} \partial_{x}\left(F_{0}^{+}+F_{0}^{-}\right) e^{i S / \varepsilon} .
\end{aligned}
$$

We observe that when $E$ is a constant, the phase $S$ does not depend on $x$.

Note that in this context, we can solve explicitly the equation on the phase $S$

$$
\partial_{t} S-E(x) \partial_{p_{x}} S=2|p|, \quad S\left(0, x, p_{x}, p_{y}\right)=0 .
$$

Using the method of the characteristics, we get

$$
\frac{d}{d t}\left[S\left(t, x, p_{x}-E t, p_{y}\right)\right]=2 \sqrt{\left(p_{x}-E t\right)^{2}+p_{y}^{2}},
$$

and integrating on $[0, t]$ leads to

$$
S\left(t, x, p_{x}-E t, p_{y}\right)-S\left(0, x, p_{x}, p_{y}\right)=2 \int_{0}^{t} \sqrt{\left(p_{x}-E s\right)^{2}+p_{y}^{2}} d s=2 \frac{p_{y}^{2}}{E} \int_{-p_{x} / p_{y}}^{\left(E t-p_{x}\right) / p_{y}} \sqrt{1+u^{2}} d u .
$$

From the relation $\int \sqrt{1+u^{2}} d u=\frac{1}{2} \xi(u)$ with $\xi(u)=u \sqrt{1+u^{2}}+\ln \left(u+\sqrt{1+u^{2}}\right)$, we finally obtain

$$
S\left(t, x, p_{x}, p_{y}\right)=\frac{p_{y}^{2}}{E}\left[-\xi\left(\frac{p_{x}}{p_{y}}\right)+\xi\left(\frac{p_{x}+E t}{p_{y}}\right)\right] .
$$




\section{References}

[1] C. W. Beenakker, Colloquium: Andreev reflection and Klein tunneling in graphene, Rev. Mod. Phys. 80 (2008), p. 1337.

[2] M. Bennoune, M. Lemou, L. Mieussens, Uniformly stable numerical schemes for the Boltzmann equation preserving the compressible Navier-Stokes asymptotics, J. Comp. Phys. 227 (2008), pp. 3781-3803.

[3] A. H. Castro Neto, F. Guinea, N. M. R. Peres, K. S. Novoselov, A. K. Geim, The electronic properties of graphene, Rev. Mod. Phys. 81 (2009), pp. 109-162.

[4] P. Chartier, N. Crouseilles, M. Lemou, F. Méhats, Uniformly accurate numerical schemes for highly oscillatory Klein-Gordon and nonlinear Schrödinger equations, Numer. Math. 129 (2015), pp. 211-250.

[5] P. Chartier, M. Lemou, F. Méhats, G. Vilmart, A new class of uniformly accurate numerical schemes for highly oscillatory evolution equations, 2017, hal-01666472.

[6] N. Crouseilles, S. Jin and M. Lemou, Nonlinear geometric optics method based multi-scale numerical schemes for a class of highly-oscillatory transport equations, Math. Model Methods Applied Sci. 27 (2017), pp. 2031-2070.

[7] N. Crouseilles, S. Jin, M. Lemou and L. Liu,, Nonlinear Geometric Optics Based Multiscale Stochastic Galerkin Methods for Highly Oscillatory Transport Equations with Random Inputs, preprint (2017).

[8] N. Crouseilles, M. Lemou, F. Méhats, Asymptotic preserving schemes for highly oscillatory kinetic equations, J. Comput. Phys. 248 (2013), pp. 287-308.

[9] N. Crouseilles, M. Lemou, F. Méhats, X. Zhao, Uniformly accurate Particle-In-Cell method for the long time solution of the two-dimensional Vlasov-Poisson equation with uniform strong magnetic field, to appear in J. Comput. Phys..

[10] A. Faraj, S. Jin, The Landau-Zener transition and the surface hopping method for the 2D Dirac equation for graphene, Commun. Comput. Phys. 21 (2017), pp. 313-357.

[11] C. Fefferman, M.I. Weinstein, Honeycomb lattice potentials and Dirac points, J. American Mathematical Society 25 (2012), pp. 1169-1220.

[12] C. Fefferman, M.I. Weinstein, Wave packets in honeycomb structures and two-dimensional Dirac equations, Comm. Math. Phys. 326 (2014), pp 251-286.

[13] G. Fiori, G. Iannaccone, Simulation of graphene nanoribbon field-effect transistors, IEEE Electron. Device Lett. 28 (2007), pp. 760-762.

[14] E. Hairer, C. Lubich, G. Wanner, Geometric Numerical Analysis: Structure-Preserving Algorithms for Ordinary Differential Equations, Springer Series in Computational Mathematics 31, Springer-Verlag, Berlin, 2nd ed., 2006.

[15] S. Jin, P. A. Markowich, C. Sparber, Mathematical and computational methods for semiclassical Schrödinger equations, Acta Numerica 20 (2011), pp. 121-209.

[16] C.F. Kammerer, F. Méhats, A kinetic model for the transport of electrons in a graphene layer, J. Comp. Phys. 327 (2016), pp. 450-483.

[17] A. Klar and C. Schmeiser, Numerical passage from radiative heat transfer to nonlinear diffusion models, Math. Models Methods Appl. Sci. 11 (2001), pp. 749-767.

[18] M. C. Lemme, T. J. Echtermeyer, M. Baus, H. Kurz, A graphene field-effect device, IEEE Electron. Device Lett. 28 (2007), pp. 282-284.

[19] M. Lemou, L. Mieussens, A new asymptotic preserving scheme based on micro-macro formulation for linear kinetic equations in the diffusion limit, SIAM J. Sci. Comp. 31 (2008), pp. 334-368.

[20] O. Morandi, F. Schürrer, Wigner model for quantum transport in graphene, J. Phys. A: Math. Theor. 44 (2012), pp. 265-301.

[21] D. S. Novikov, Elastic scattering theory and transport in graphene, Phys. Rev. B 76 (2007), 245435.

[22] K. S. Novoselov, A. K. Geim, S. V. Morozov, D. Jiang, Y. Zhang, S. V. Dubonos, I. V. Gregorieva, A. A. Firsov, Electric field effect in atomically thin carbon films, Science 306 (2004), pp. 666-669. 